\title{
MONSTER IN THE DARK: THE ULTRALUMINOUS GRB 080607 AND ITS DUSTY ENVIRONMENT
}

\author{
D. A. Perley ${ }^{1}$, A. N. Morgan ${ }^{1}$, A. Updike $^{2}$, F. Yuan ${ }^{3}$, C. W. Akerlof ${ }^{3}$, A. A. Miller ${ }^{1}$, J. S. Bloom ${ }^{1}$, S. B. Cenko $^{1}$, W. Li ${ }^{1}$, \\ A. V. Filippenko ${ }^{1}$, J. X. Prochaska ${ }^{4}$, D. A. KanN ${ }^{5}$, N. R. Tanvir ${ }^{6}$, A. J. Levan ${ }^{7}$, N. R. Butler ${ }^{1}$, P. Christian ${ }^{1}$, \\ D. H. Hartmann ${ }^{2}$, P. Milne ${ }^{8}$, E. S. RykofF ${ }^{9}$, W. Rujopakarn ${ }^{8}$, J. C. Wheeler ${ }^{10}$, And G. G. Williams ${ }^{8}$ \\ ${ }^{1}$ Department of Astronomy, University of California, Berkeley, CA 94720-3411, USA; dperley@ astro.berkeley.edu \\ ${ }^{2}$ Department of Physics and Astronomy, Clemson University, Clemson, SC 29634-0978, USA \\ ${ }^{3}$ University of Michigan, Randall Laboratory of Physics, 450 Church Street, Ann Arbor, MI 48109-1040, USA \\ ${ }^{4}$ Department of Astronomy and Astrophysics, UCO/Lick Observatory, University of California, 1156 High Street, Santa Cruz, CA 95064, USA \\ ${ }^{5}$ Thuringer Landessternwarte Tautenburg, Sternwarte 5, D-07778 Tautenburg, Germany \\ ${ }^{6}$ Department of Physics and Astronomy, University of Leicester, University Road, Leicester LE1 7RH, UK \\ ${ }^{7}$ Department of Physics, University of Warwick, Coventry CV4 7AL, UK \\ ${ }^{8}$ Steward Observatory, University of Arizona, 933 North Cherry Avenue, Tucson, AZ 85721, USA \\ ${ }^{9}$ Physics Department, University of California at Santa Barbara, 2233B Broida Hall, Santa Barbara, CA 93106, USA \\ ${ }^{10}$ Department of Astronomy, University of Texas, Austin, TX 78712, USA \\ Received 2010 August 31; accepted 2010 October 28; published 2010 December 29
}

\begin{abstract}
We present early-time optical through infrared photometry of the bright Swift gamma-ray burst (GRB) 080607, starting only $6 \mathrm{~s}$ following the initial trigger in the rest frame. Complemented by our previously published spectroscopy, this high-quality photometric data set allows us to solve for the extinction properties of the redshift 3.036 sightline, giving perhaps the most detailed information to date on the ultraviolet continuum absorption properties of any sightline outside our Local Group. The extinction properties are not adequately modeled by any ordinary extinction template (including the average Milky Way, Large Magellanic Cloud, and Small Magellanic Cloud curves), partially because the $2175 \AA$ feature (while present) is weaker by about a factor of two than when seen under similar circumstances locally. However, the spectral energy distribution is exquisitely fitted by the more general Fitzpatrick \& Massa parameterization of Local-Group extinction, putting it in the same family as some peculiar Milky Way extinction curves. After correcting for this (considerable, $A_{V}=3.3 \pm 0.4 \mathrm{mag}$ ) extinction, GRB 080607 is revealed to have been among the most optically luminous events ever observed, comparable to the naked-eye burst GRB 080319B. Its early peak time $\left(t_{\text {rest }}<6 \mathrm{~s}\right)$ indicates a high initial Lorentz factor $(\Gamma>600)$, while the extreme luminosity may be explained in part by a large circumburst density. Only because of its early high luminosity could the afterglow of GRB 080607 be studied in such detail in spite of the large attenuation and great distance, making this burst an excellent prototype for the understanding of other highly obscured extragalactic objects, and of the class of "dark" GRBs in particular.
\end{abstract}

Key words: dust, extinction - gamma-ray burst: individual (080607)

Online-only material: color figures, machine-readable and VO tables

\section{INTRODUCTION}

The most extreme gamma-ray bursts (GRBs) have often been the most illuminating-both literally and figuratively. The enormous isotropic-equivalent energy of GRB 971214 (redshift $z=3.43, E_{\text {iso }}=3 \times 10^{53}$ erg; Ramaprakash et al. 1998; Odewahn et al. 1998; Kulkarni et al. 1998) emphatically demonstrated the need for collimation to bring the energy budget of long-duration GRBs within physically reasonable values. Observations of the mag 9 optical flash of GRB 990123 $\left(z=1.61, E_{\text {iso }}=3.4 \times 10^{54} \mathrm{erg}\right.$; Akerlof et al. 1999 ; Kulkarni et al. 1999) anticipated the utility of GRBs to probe the highredshift universe: similar events would be easily detectable even at $z>6$. This possibility was first vindicated by GRB 050904 $\left(z=6.29, E_{\text {iso }}=1.2 \times 10^{54} \mathrm{erg}\right.$; Kawai et al. 2006; Sugita et al. 2008), which for three years remained the most distant GRB known and, at the time, was also the most luminous optical transient observed in the universe (Kann et al. 2007). The latter record has since been surpassed dramatically by GRB 080319B $\left(z=0.937, E_{\text {iso }}=1.3 \times 10^{54} \mathrm{erg}\right)$, whose optical afterglow peaked at $V \approx 5 \mathrm{mag}$ (Racusin et al. 2008; Bloom et al. 2009; Woźniak et al. 2009). The current record for the bolometric isotropic-equivalent energy is held by the Fermi burst GRB
080916C $\left(E_{\text {iso }}=6.5 \times 10^{54} \mathrm{erg}\right.$; Abdo et al. 2009a; Greiner et al. 2009).

Joining this list of record setters is GRB $080607(z=3.036$; Prochaska et al. 2009), with $E_{\text {iso }}=1.87 \times 10^{54} \mathrm{erg}$ (Golenetskii et al. 2008). This event is remarkable not only for its intrinsic properties, but also because of its unusual environment: a Keck spectrum obtained starting only 20 minutes after the burst (Prochaska et al. 2009) reveals that the sightline penetrates a giant molecular cloud in the host galaxy, obscuring the restframe visible light by $A_{V} \approx 3$ mag of extinction (or $\sim 6$ mag at $1600 \AA$, corresponding to the observed $R$ band) before it even began its journey through intergalactic space. ${ }^{11}$ In spite of this extreme attenuation, the event was bright enough to be detected by small optical telescopes for over an hour.

The spectroscopic properties of this event have been previously discussed by Prochaska et al. (2009), along with a preliminary analysis of its extinction properties; further analysis of the spectra was also presented by Sheffer et al. (2009). In this paper, we analyze several other aspects of this burst, from the

\footnotetext{
11 Dust extinction is limited or absent for the vast majority of well-studied GRBs (Schady et al. 2007; Kann et al. 2010), and no other GRB displays firm evidence for molecular lines.
} 
prompt emission (and simultaneous optical detection) through a late-time search for the host galaxy, and we present a significantly expanded discussion of its extinction properties. In Section 2, we describe our early-time multicolor observations of the afterglow with several different robotic telescopes. We analyze the optical light curve in Sections 3.1 and 3.2 and show no correlation between the prompt-emission behavior and the early optical observations, starting at only $6 \mathrm{~s}$ post-trigger in the host frame, and we present limits on color variations at early times. In Sections 3.3 and 3.4, we examine in more detail the combined photometric and spectroscopic spectral energy distribution (SED) and place our final constraints on the hostgalaxy extinction properties, demonstrating the firm detection of a $2175 \AA$ bump, the highest-redshift detection of this signature to date. The X-ray light curve is analyzed in Section 3.5 to search for evidence of dust scattering in the host at these wavelengths. In Sections 4 and 5, we place GRB 080607 and its environment in the context of other GRBs, both ultraluminous and bright events like GRB 080319B as well as the poorly understood class of extremely dark bursts (Jakobsson et al. 2004).

\section{OBSERVATIONS}

\subsection{Swift}

GRB 080607 triggered the Burst Alert Telescope (BAT; Barthelmy et al. 2005) on the Swift satellite (Gehrels et al. 2004) at 06:07:27 on 2010 June 7 (UT dates are used throughout this paper; times are referenced to this trigger time, although it is important to note that there was significant emission before this trigger). The light curve (Figure 1) is spiky and erratic, exhibiting a dominant peak at $\sim 4 \mathrm{~s}$ as well as numerous other, fainter peaks ranging from a few seconds before the trigger out to $\sim 130 \mathrm{~s}$ after, when the signal falls below the background level. Swift slewed immediately to the source and began pointed observations with the X-ray Telescope (XRT; Burrows et al. 2005) at $82 \mathrm{~s}$, followed by observations with the Ultraviolet Optical Telescope (UVOT; Roming et al. 2005) beginning at 100 s. Observations continued until 1049 s, after which Swift slewed away temporarily, returning to the field at 4226 s. From then, observations continued intermittently over the next four days, after which the X-ray flux was too faint for Swift to detect.

The BAT observations were processed using the Swift HEAsoft 6.5 software package via the burst pipeline script, batgrbproduct. We calculated spectral parameters both directly and using the Bayesian formalism described by Butler et al. (2007). Fitted to this burst alone, a Band et al. (1993) model provides no significant improvement over a basic power-law fit over BAT's 15-350 keV energy range (photon index $\Gamma=1.16$ ), suggesting a peak energy above the BAT range. Using the Band model Bayesian estimate of $E_{\text {peak,obs }}=902_{-460}^{+1170} \mathrm{keV}$ and the measured redshift $(z=3.036)$, we estimate a broadband isotropic-equivalent energy of $E_{\text {iso }}=2.8_{-0.9}^{+1.3} \times 10^{54} \mathrm{erg}$. These values place GRB 080607 second in $E_{\text {iso }}$ rank among all Swift GRBs to date and in the same regime as extreme GRBs 080319B and 990123.

GRB 080607 was observed by other satellites as well (Konus-Wind and Super-AGILE), enabling a precise measurement of the spectral parameters. An in-depth analysis of the Konus data will be presented in future work by Sbarufatti et al., but preliminary calculations from Golenetskii et al. (2008) give the following values: $E_{\text {peak,obs }}=394_{-54}^{+58} \mathrm{keV}$ and $E_{\text {iso }}=1.87_{-0.10}^{+0.11} \times 10^{54} \mathrm{erg}$. These are at the low end of, but generally consistent with, the Bayesian Swift result, and confirm

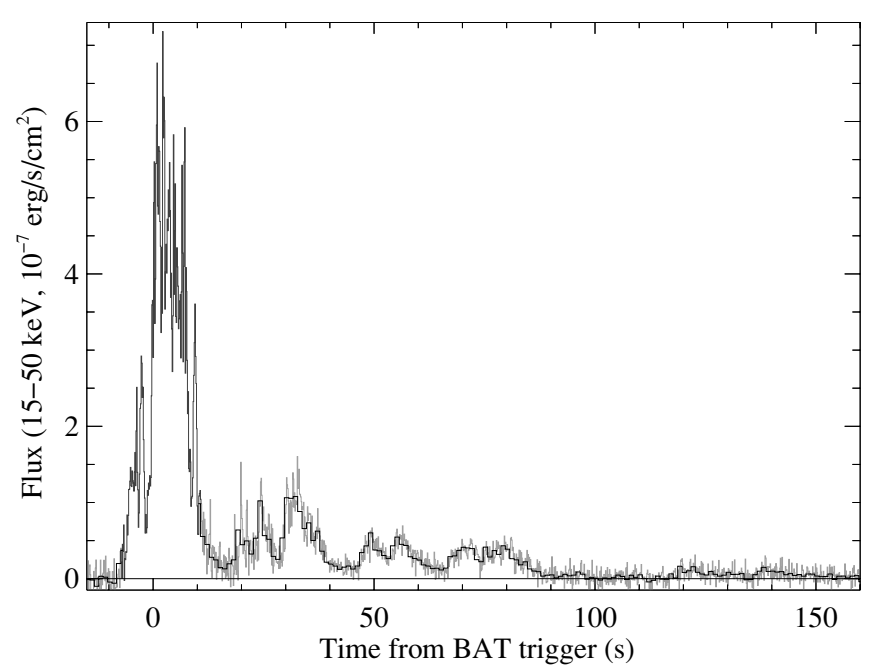

Figure 1. Gamma-ray light curve of GRB 080607 (at a combination of $128 \mathrm{~ms}$ and $1 \mathrm{~s}$ binning) showing the bright initial pulse complex followed by an additional series of pulses lasting for the next several minutes. Original data for the light curve are taken from the Swift Burst Analyzer (Evans et al. 2010).

that GRB 080607 was among the most luminous and intrinsically hardest (highest $E_{\text {peak,rest }}$ ) GRBs observed by any satellite.

The X-ray afterglow was detected throughout the XRT observations; XRT data were reduced by the procedures of Butler \& Kocevski (2007). The UVOT afterglow, by contrast, is only marginally detected in the earliest epoch, and only in White and $V$ filters (Schady et al. 2008). Both filters are heavily impacted by damped $\operatorname{Ly} \alpha$ absorption at $z=3.036$, and so are not used in our analysis.

\subsection{ROTSE}

The ROTSE-III (Robotic Optical Transient Search Experiment) array is a worldwide network of $0.45 \mathrm{~m}$ robotic, automated telescopes, built for fast responses to GRB triggers (Akerlof et al. 2003). ROTSE-IIIb, located at the McDonald Observatory, Texas, responded immediately to the initial Gamma-ray Burst Coordinate Network (GCN; Barthelmy et al. 1995) alert. The first image started at 06:07:49.0 (22 s after the burst), clearly detecting a bright afterglow at the XRT position in this exposure. All ROTSE-III images were processed with our custom RPHOT photometry program based on the DAOPHOT (Stetson 1987) point-spread function fitting photometry package (Quimby et al. 2006). The unfiltered, thinned ROTSE-III CCD has a peak sensitivity in the wavelength range of the $R$ band. The ROTSE magnitudes were thus adjusted using the median offset from the USNO B1.0 $R$-band measurements of selected field stars. Observations are presented (along with photometry from all other telescopes, below) in Table 1 .

\subsection{Super-LOTIS}

Super-LOTIS (Livermore Optical Transient Imaging System) is a robotic $0.6 \mathrm{~m}$ telescope dedicated to the search for optical counterparts of GRBs (Williams et al. 2004, 2008). The telescope is housed in a roll-off-roof facility at the Steward Observatory Kitt Peak site near Tucson, AZ. Super-LOTIS triggered on GRB 080607 and began observations at 06:08:03 (36 s after the trigger) acquiring a series of frames in the $R$ band. The images were reduced and photometry performed using standard techniques, calibrated relative to nearby Sloan Digital Sky Survey (SDSS) standard stars. 
Table 1

Photometry of GRB 080607

\begin{tabular}{lccccc}
\hline \hline Telescope/GCN & $\begin{array}{c}t^{\mathrm{a}} \\
(\mathrm{s})\end{array}$ & Filter & $\begin{array}{c}\text { Exp. Time } \\
(\mathrm{s})\end{array}$ & Mag. $^{\mathrm{b}}$ & $\begin{array}{c}\text { Flux }^{\mathrm{c}} \\
(\mu \mathrm{Jy})\end{array}$ \\
\hline PAIRITEL & 89.0 & $J$ & 23.4 & $13.766 \pm 0.107$ & $5048.6 \pm 475.4$ \\
PAIRITEL & 89.0 & $H$ & 23.4 & $12.050 \pm 0.109$ & $15657.3 \pm 1501.3$ \\
PAIRITEL & 89.0 & $K_{s}$ & 23.4 & $10.750 \pm 0.139$ & $33681.7 \pm 4058.1$ \\
KAIT & 188.0 & Clear & 20.0 & $17.501 \pm 0.055$ & $326.0 \pm 16.1$ \\
KAIT & 158.0 & $I$ & 20.0 & $16.582 \pm 0.094$ & $587.2 \pm 48.7$ \\
KAIT & 128.0 & $V$ & 20.0 & $17.538 \pm 0.142$ & $379.9 \pm 46.6$ \\
ROTSE & 24.5 & Clear & 5.0 & $14.920 \pm 0.040$ & $3512.1 \pm 127.0$ \\
Super-LOTIS & 40.8 & $R$ & $\ldots$ & $15.060 \pm 0.059$ & $3087.2 \pm 163.7$ \\
P60 & 234.8 & $R$ & 60.0 & $17.524 \pm 0.014$ & $319.1 \pm 4.1$ \\
P60 & 406.1 & $i$ & 60.0 & $18.359 \pm 0.022$ & $171.1 \pm 3.4$ \\
P60 & 491.8 & $z$ & 60.0 & $18.694 \pm 0.092$ & $127.3 \pm 10.3$ \\
UKIRT & 2347.5 & $K$ & 360.0 & $14.624 \pm 0.025^{\mathrm{d}}$ & $949.7 \pm 21.6^{\mathrm{d}}$ \\
UKIRT & 4231.5 & $J$ & 180.0 & $18.470 \pm 0.054$ & $66.29 \pm 3.22$ \\
UKIRT & 4524.0 & $H$ & 180.0 & $17.060 \pm 0.035$ & $155.1 \pm 4.9$ \\
\hline
\end{tabular}

Notes. Contains only the first data point in each filter taken by each telescope.

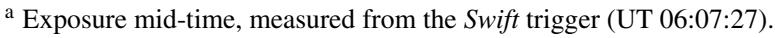

b Observed value, not corrected for Galactic extinction.

${ }^{\mathrm{c}}$ Corrected for Galactic extinction $\left(E_{B-V}=0.023 \mathrm{mag}\right)$.

d Point not used in modeling.

(This table is available in its entirety in machine-readable and Virtual Observatory (VO) forms in the online journal. A portion is shown here for guidance regarding its form and content.)

\subsection{KAIT}

The Katzman Automatic Imaging Telescope (KAIT) at Lick Observatory ( $\mathrm{Li}$ et al. 2003) also responded automatically to the Swift alert and began taking observations, the first starting at 06:09:25, $118 \mathrm{~s}$ after the BAT trigger. The KAIT filter sequence consists of a series of unfiltered observations, followed by a cycle through $V, I$, and unfiltered exposures. The optical afterglow was detected in all filters, although it is quite faint in the $V$ band. Following this sequence, a series of unfiltered and $I$-band exposures was manually added, although the afterglow was not detected in the $I$ band and only marginally detected in our unfiltered exposures at that time (even after stacking).

Images were reduced using standard techniques. This left some residual variation in the background sky, which was removed by subtraction of an illumination frame. We used aperture photometry to measure the afterglow flux, calibrating relative to SDSS stars in the field transformed to the Johnson/Cousins system using the Lupton (2006) transformation equations ${ }^{12}$. The unfiltered exposures were calibrated to the $R$ band ( $\mathrm{Li}$ et al. 2003).

\subsection{PAIRITEL}

The robotic Peters Automatic Infrared Imaging Telescope (PAIRITEL; Bloom et al. 2006) consists of the $1.3 \mathrm{~m}$ Peters Telescope at Mt. Hopkins, AZ-formerly used for the Two Micron All Sky Survey (2MASS; Skrutskie et al. 2006)—refurbished with the southern 2MASS camera. PAIRITEL uses two dichroics to image in the infrared (IR) $J, H$, and $K_{S}$ filters simultaneously every $7.8 \mathrm{~s}$.

PAIRITEL responded to the initial BAT alert and slewed immediately to the source. Observations began at 06:08:44, $77 \mathrm{~s}$ after the trigger, and continued for the next $1.3 \mathrm{hr}$ until the source reached its hour-angle limit.

\footnotetext{
12 http://www.sdss.org/dr7/algorithms/sdssUBVRITransform.html
} \#Lupton2005.
The early-time $(<0.3 \mathrm{hr})$ raw data files were processed using standard IR reduction methods via PAIRITEL Pipeline III (C. Klein et al. 2011, in preparation) and resampled using SWarp (Bertin et al. 2002) to create $1^{\prime \prime} .0$ pixel $^{-1}$ images for final photometry. Due to changing sky conditions that complicated the otherwise superior Pipeline III reductions in the $K_{s}$ band as the source approached the horizon, the remainder of the raw data were reduced using an older pipeline which utilized a "dark bank" that more robustly handles flat-fielding in such cases.

PAIRITEL's standard observing cycle is to take three $7.8 \mathrm{~s}$ exposures in immediate succession at each dither position. While the early afterglow is detected in even the shortest $7.8 \mathrm{~s}$ frames, for signal-to-noise ratio $(\mathrm{S} / \mathrm{N})$ and calibration considerations, we report $23.4 \mathrm{~s}$ "triplestacks" (the median of all three images at each dither position) as our shortest exposures. These images were further binned at successively later times to further improve the $\mathrm{S} / \mathrm{N}$.

Aperture photometry was performed using custom Python software, utilizing Source Extractor (SExtractor; Bertin \& Arnouts 1996) as a back end. Four calibration stars present in all images were chosen based on brightness, proximity of nearby contaminating sources, and location relative to bad pixels. The optimal aperture of $\sim 3^{\prime \prime}$ radius was determined by minimizing the absolute error relative to 2MASS magnitudes of our four calibration stars.

Calibration was performed by redetermining the zero point for each image individually by comparison to 2MASS magnitudes using these four stars. The resulting statistical uncertainty in the zero point is negligible relative to other sources of error. Additional, systematic sources of error are addressed in detail by Perley et al. (2010); we use a similar procedure here to determine the total uncertainty of each point.

\section{6. $P 60$}

The robotic Palomar 60 inch telescope (P60; Cenko et al. 2006) automatically responded to the Swift trigger for GRB 080607, executing a predefined sequence of observations 
in the Kron $R$ and Sloan $i^{\prime}$ and $z^{\prime}$ filters beginning $174 \mathrm{~s}$ after the burst trigger time. Individual images were reduced in real time using standard IRAF ${ }^{13}$ routines. The images were calibrated with respect to several dozen field stars from the SDSS Data Release 7 (Abazajian et al. 2009), using the filter transformations of Jordi et al. (2006) for the Kron $R$ filter.

\subsection{UKIRT}

The United Kingdom Infrared Telescope (UKIRT) began observations of GRB 080607 starting at approximately 39 minutes after the GRB trigger. An initial series of three $K$-band exposures was acquired, followed by a $J H J K$ sequence, repeated three times. The afterglow is detected at very high significance $(>10 \sigma)$ in all co-added frames.

Observations were reduced using standard IR techniques and calibrated relative to 2MASS standards in the field. In the case of the $K$ observations, we convert $K$ to $K_{s}$ using color terms derived from bright 2MASS standards in the field, but because the afterglow is extremely red and the field stars are all much bluer (with very limited dispersion in color), this term is quite uncertain, and the $K$ observations are not used in fitting.

The telescope was dithered only once during the exposure sequence (after the first three $K$-band exposures). A pixelsampling uncertainty $(0.025 \mathrm{mag}$, derived from the median absolute offset of bright stars before and after the dither) was added in quadrature to all data points, but it should be noted that this uncertainty is correlated (points before and after the dither are all affected in nearly the same way; indeed, a small shift is evident between the first and last three $K$-band exposures.)

Although we do not find significant evidence of color change for this GRB afterglow (Section 3.1), given that the UKIRT points are nonsimultaneous with all other filters, we do not include them when deriving the broadband SED used for modeling (Section 3.3). On the other hand, we do use them to constrain the late-time evolution of the light curve.

\subsection{Keck Spectroscopy}

We initiated spectroscopic observations of the afterglow with the Low Resolution Imaging Spectrometer (LRIS; Oke et al. $1995)$ on the Keck I 10 m telescope at 13 minutes after the Swift trigger, although due to poor guiding this first frame was not usable. The first exposure used in our analysis began at 20.1 minutes following the trigger. Several additional exposures were taken over the next $2 \mathrm{hr}$ using the B600 grism and both the R400 and R1200 gratings; our final observations span a wavelength range of 3000-9000 $\AA$. Observations were flux calibrated relative to the spectroscopic standard HZ 44. More details on these spectroscopic observations and our reductions are given by Prochaska et al. (2009).

\subsection{Keck Host-galaxy Imaging}

The field of GRB 080607 was imaged in several deep integrations at Keck through various optical/IR filters $(g, I$, and $\left.K_{s}\right)$. None of these integrations resulted in a secure detection of the host galaxy, although marginal $(1 \sigma-3 \sigma)$ detections of flux above the background level are present in the $g$ and $K_{s}$ observations. A log of our ground-based host observations is reported in Table 2 .

\footnotetext{
13 IRAF is distributed by the National Optical Astronomy Observatory, which is operated by the Association for Research in Astronomy, Inc., under cooperative agreement with the National Science Foundation (NSF).
}

Table 2

Host-galaxy Limits

\begin{tabular}{lcccc}
\hline \hline Instrument & $\begin{array}{c}\text { Obs. Date } \\
(\mathrm{UT})\end{array}$ & $\begin{array}{c}\text { Exp. Time } \\
(\mathrm{s})\end{array}$ & Filter & $\begin{array}{c}5 \sigma \text { Limit } \\
(\mathrm{mag})\end{array}$ \\
\hline Keck I/LRIS & 2009 Feb 19 & 2490 & $g$ & $>27.3$ \\
Keck I/LRIS & 2009 Feb 19 & 2220 & $I$ & $>25.3$ \\
Keck I/NIRC & 2009 May 31 & 3600 & $K_{s}$ & $>21.6$ \\
\hline
\end{tabular}

Notes. $5 \sigma$ limiting magnitudes on a host galaxy at the afterglow position from our ground-based optical and IR observations at the Keck Observatory. Magnitudes are in the SDSS $(g)$, Vega $(I)$, or 2MASS $\left(K_{s}\right)$ systems and not corrected for Galactic extinction.

The host galaxy is, however, well detected at $1.6 \mu \mathrm{m}$ in a deep Hubble Space Telescope image using WFC3, as well as in both of the warm Spitzer IRAC channels $(3.6 \mu \mathrm{m}$ and $4.5 \mu \mathrm{m})$. The extreme optical faintness of this system, while partially due simply to its high redshift $(z=3.036)$, makes this galaxy of particular interest: determination of the redshift would be exceptionally difficult using traditional field-survey techniques, illustrating the unique ability of GRBs to select and study optically faint galaxies at high redshift. Its red color, likely reflective of large internal dust content, is also noteworthy. Further discussion of the host galaxy, including detailed analysis of both the ground- and space-based imaging, is presented by Chen et al. (2010).

\section{ANALYSIS}

\subsection{Light Curve}

The multi-band light curve of GRB 080607 is plotted in Figure 2. After an initially slow decay, the light curve steepens (decay index $\alpha=1.6$, using the convention $F \propto t^{-\alpha}$ ) before flattening out at $1000 \mathrm{~s}$ to a temporarily flat decay. This slow decay lasts for approximately another hour before fading rapidly, falling below the detection threshold of subsequent KAIT images, but still well detected by UKIRT.

The light curve was fitted using the techniques described by Perley et al. (2010) and previous works by our group, modeling the light curve as the sum of several broken power laws. Our temporal coverage of this event is limited (ending at $10^{4} \mathrm{~s}$ ), making the analysis simple: we employ two Beuermann et al. (1999) broken power laws, one to describe the early behavior and the second to describe the later flattening. Because we do not detect the rising phase of the afterglow, the pre-break index of the first power-law component is not usefully constrained by our data and is fixed arbitrarily to -0.5 . The X-ray light curve (after $130 \mathrm{~s}$ ) was fitted using similar techniques, but with unbroken power laws. We do not attempt to model the low-level late-time X-ray flaring that appears to be present in the data.

Modest but significant color change has been previously observed in early-time GRB afterglows (see Perley et al. 2008; Bloom et al. 2009 for two prominent examples), a possibility which we model by allowing the intrinsic spectral power-law index $\beta\left(F \propto v^{-\beta}\right)$ to vary between components or across breaks. However, in the case of GRB 080607, any such color change is not significant: the change in the intrinsic index between the fast-decay and flat components is just $\Delta \beta=$ $0.05 \pm 0.07$ and only modestly improves the goodness of fit. Therefore, for simplicity we assume no color change during our observations of this burst.

Because of the relatively short temporal coverage and lack of overlap between the X-ray and optical light curves (except at the 


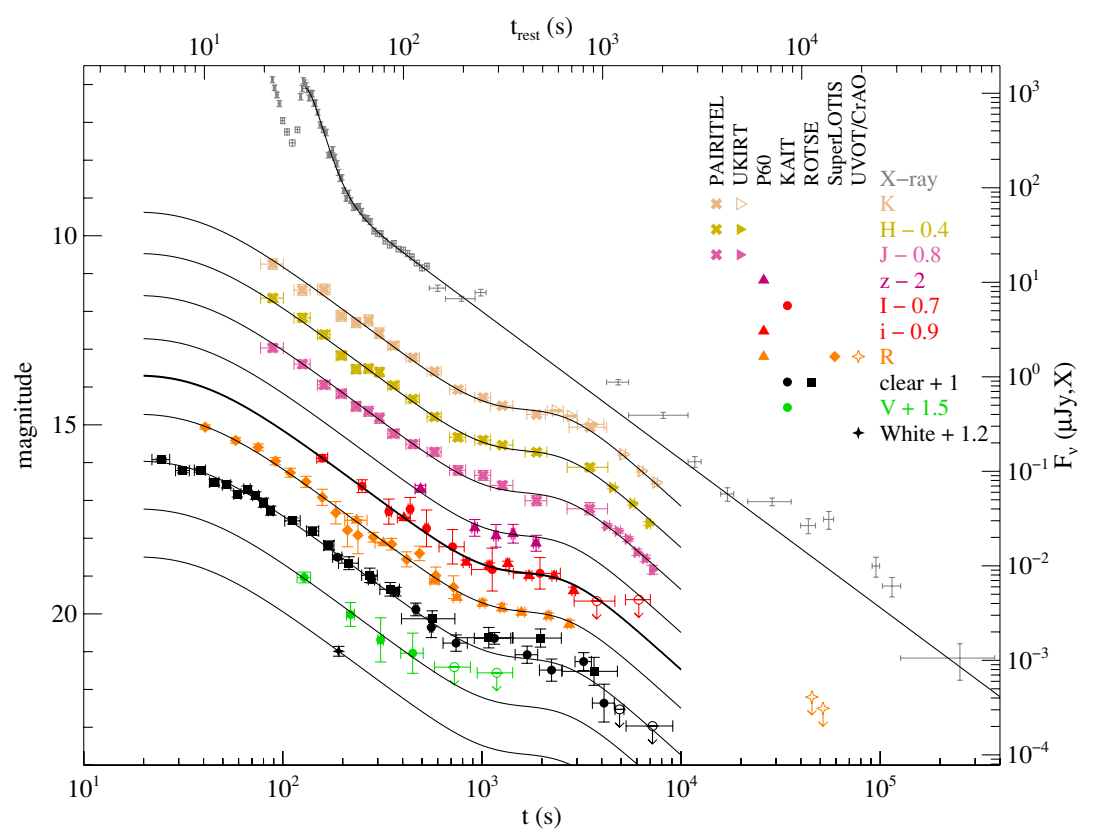

Figure 2. Multi-band light curve of GRB 080607 from a variety of ground-based telescopes as well as the Swift XRT fitted to a sum of two broken power laws. (XRT data are fitted to a sum of two unbroken power laws.) Magnitudes are in the Vega (VRI), SDSS ( $r i)$, or 2MASS (JHK) systems and (with the exception of $R$ and $K_{s}$ ) have been shifted as indicated for clarity; these magnitudes are not corrected for Galactic extinction (which is nearly insignificant) or host-galaxy extinction (which is very large). The afterglow initially fades slowly, then steepens; it briefly levels out at $10^{3} \mathrm{~s}$ before breaking again and is not detected after $5000 \mathrm{~s}$. The late-time $R$-band limits are from Rumyantsev \& Pozanenko (2008). We use the BAT trigger time for $t_{0}$ (06:07:27 on 2010 June 7), which corresponds to the start of the largest prompt-emission pulse; using the start of gamma-ray emission instead does not significantly change the qualitative results.

(A color version of this figure is available in the online journal.)

earliest times; see Section 3.2), it is difficult to unambiguously associate the features of the light curve with intrinsic properties of the burst itself. The moderately rapid early decay $\left(\alpha_{\mathrm{opt}} \approx\right.$ $\left.\alpha_{\mathrm{X}} \approx 1.6\right)$ is suggestive of a reverse shock or possibly a wind-driven medium (see also Section 4.2), although we cannot distinguish between these cases with the data available, and a constant-density environment is also plausible if both optical and X-ray bands are above the cooling break. The achromatic flattening (and subsequent rapid falloff) may be produced by a density variation, energy reinjection episode, or other feature. Interestingly, the X-ray flux increases significantly just before the end of the first-orbit observations (and just before the start of the optical flattening), but because the flattening itself corresponds to the Swift orbit gap it is difficult to determine whether the two features share a common origin.

\subsection{Absence of Optical/High-energy Correlations}

Our optical follow-up observations of this burst begin extremely early. The ROTSE coverage begins at only $21 \mathrm{~s}$ after the BAT trigger, corresponding to less than $6 \mathrm{~s}$ in the GRB rest frame. The prompt emission was still extremely active at this time: at least five major gamma-ray flares occurred during our optical observations, the last of which was also caught at X-ray wavelengths by the XRT. PAIRITEL, KAIT, and Super-LOTIS were all observing during this last flare.

Even in this rich overlapping data set, there is no correlation visible between the optical and high-energy light curves of the type seen by, for example, Vestrand et al. (2005), Blake et al. (2005), and Beskin et al. (2010). In Figure 3, we overplot the gamma-ray, X-ray, and optical light curves using the same relative scaling. In spite of the erratic, flaring high-energy behavior, we see no sign of significant deviation of the optical light curves from their smooth power-law behavior at any point.

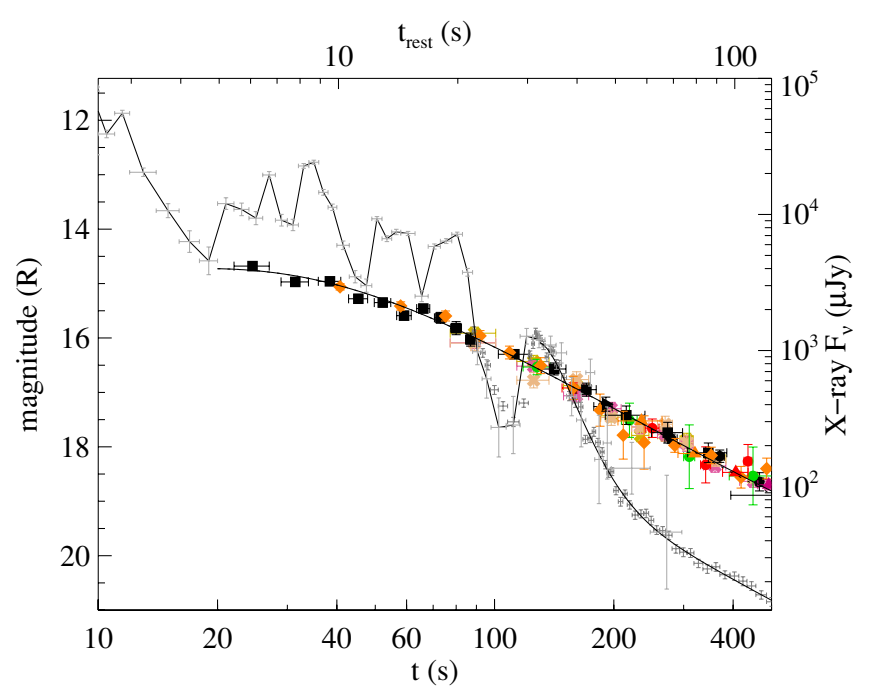

Figure 3. Early-time optical and gamma-ray/X-ray light curves of GRB 080607 demonstrating the extremely early peak $t_{\text {rest }} \lesssim 6 \mathrm{~s}$ as well as the absence of any visible correlation between the optical and high-energy light curves. (Optical fluxes in other bands have been scaled to match the $R$ band; the gamma-ray light curve is scaled to match the X-ray curve.) Symbols are the same as in Figure 2. (A color version of this figure is available in the online journal.)

This is consistent with other ROTSE-followed bursts (e.g., Yost et al. 2007; Rykoff et al. 2009) and provides another clear example of a burst whose afterglow behavior is clearly divorced from that of the prompt emission.

The lack of even modest influence of the prompt emission on the afterglow may initially seem surprising: even if truly prompt (internal-shock) emission is absent in this band, one might expect that some of the energy being released so liber- 
Table 3

Model Fluxes at $t=300 \mathrm{~s}$

\begin{tabular}{|c|c|c|c|c|c|c|c|c|}
\hline Filter & $\begin{array}{l}\lambda_{\text {obs }} \\
(\AA)\end{array}$ & $\begin{array}{l}F_{\text {obs }} \\
(\mu \mathrm{Jy})\end{array}$ & $\begin{array}{l}m_{\mathrm{obs}} \\
(\mathrm{mag})\end{array}$ & $\begin{array}{l}A_{\lambda, \mathrm{Gal}} \\
(\mathrm{mag})\end{array}$ & $\begin{array}{c}\Delta m_{\text {lines }} \\
(\mathrm{mag})\end{array}$ & $\begin{array}{c}m_{\text {cont }} \\
(\mathrm{AB} \text { mag) }\end{array}$ & $\begin{array}{l}F_{\text {cont }} \\
(\mu \mathrm{Jy})\end{array}$ & $\begin{array}{c}A_{\lambda, \text { host }} \\
(\mathrm{mag})\end{array}$ \\
\hline X-ray & 12.4 & 34.04 & & & & & & \\
\hline$R$ & 6588 & $200.6 \pm 12.6$ & $17.97 \pm 0.07$ & 0.06 & 0.21 & $17.88 \pm 0.07$ & $256.6 \pm 16.1$ & 5.76 \\
\hline$i$ & 7706 & $260.5 \pm 16.6$ & $17.86 \pm 0.07$ & 0.05 & 0.06 & $17.75 \pm 0.07$ & $287.5 \pm 18.3$ & 5.88 \\
\hline$J$ & 12350 & $867.4 \pm 51.4$ & $15.66 \pm 0.06$ & 0.02 & 0 & $16.54 \pm 0.06$ & $883.1 \pm 52.3$ & 4.94 \\
\hline$H$ & 16620 & $2296 \pm 131$ & $14.12 \pm 0.06$ & 0.01 & 0 & $15.48 \pm 0.06$ & $2322 \pm 132$ & 4.20 \\
\hline$K_{s}$ & 21590 & $5866 \pm 337$ & $12.64 \pm 0.06$ & 0.01 & 0 & $14.47 \pm 0.06$ & $5911 \pm 339$ & 3.30 \\
\hline
\end{tabular}

Notes. Broadband afterglow fluxes as determined by the light-curve model, interpolated to $t=300 \mathrm{~s}$ after the trigger. Observed magnitudes are not corrected for Galactic extinction and are in the Vega (VRI), SDSS $(i z)$, or 2MASS (JHK $\left.K_{s}\right)$ systems. Continuum magnitudes and fluxes have been corrected for both Galactic extinction (from NED) and line absorption (calculated using our optical Keck spectroscopy).

ally by the central engine might end up in the external shock, causing a less dramatic but still observable rebrightening of the afterglow (a refreshed shock; Panaitescu et al. 1998). We note, however, that despite the intense flaring shown in Figure 3, this emission is actually dwarfed by an earlier episode: the initial pulse of the prompt emission (see Figure 1) exceeds any of the later spikes by an order of magnitude in both intensity and energy, and it is this initial pulse that dominates the energetics of the burst. The later flares are much more modest by comparison, so even presuming direct input from outflow (revealed by the prompt emission) to external shock (revealed by the afterglow), the absence of further brightening is not necessarily surprising.

\subsection{Spectral Energy Distribution}

Our light-curve fits naturally provide values for the afterglow flux in each filter at any given time, allowing us to model the SED at any time during our observations. Because of the absence of significant color change, the choice of extraction epoch is arbitrary; $300 \mathrm{~s}$ is chosen in this case (when all ground-based robotic telescopes were observing and the afterglow was still bright enough to be well detected in all bands).

The Keck optical spectroscopy covers a wide range of wavelengths and was carefully flux calibrated: photometric standard stars were observed immediately after our observations at similar airmasses and the night was photometric throughout. Accordingly, we couple our spectrum to the photometry to improve the precision of our broadband modeling.

The optical spectrum is replete with lines from a variety of elements and molecules at the host-galaxy redshift of 3.036. The analysis of these line features is discussed extensively by Prochaska et al. (2009) and Sheffer et al. (2009), and we will not repeat it here; our primary interest is in the continuum. Although the contribution of absorption lines is usually ignored in GRB photometric dust modeling, the lines in the spectrum of GRB 080607 are so abundant and so strong that ignoring them would create systematic errors significantly larger than our photometric uncertainties in both the spectrum itself and in the broadband photometry. In addition, nearly the entire spectrum at wavelengths shorter than $\sim 6900 \AA$ is affected by a forest of weak lines from vibrationally excited $\mathrm{H}_{2}^{*}$, further complicating the analysis.

Fortunately, we are able to correct for these effects. We use the line list presented in Table 1 of Prochaska et al. (2009) to identify all regions of the spectrum affected by ionic lines, including the entire spectrum blueward of $5400 \AA$, which is affected by the host damped $\operatorname{Ly} \alpha$ and the Ly $\alpha$ forest. In addition, the spectrum is corrected for the subtler but more widespread $\mathrm{H}_{2}^{*}$ absorption using the model developed by Sheffer et al. (2009). We then fit a sixth-order polynomial to the ionic line-free regions of this corrected spectrum to create a continuum model and perform synthetic photometry using both the model spectrum and the observed, uncorrected spectrum (and take the ratio) to calculate an adjustment factor with which to convert the observed (line-affected) fluxes to continuum (line-free) fluxes for each of our broadband filters covering the optical spectrum $(R, I, i$, and $V$; we assume the line contribution is small further to the red). We also wish to use the flux-calibrated spectrum itself in later analysis, so we scale the spectrum to the photometric SED extraction epoch of $300 \mathrm{~s}$ (the scale factor is determined by the value that minimizes $\chi^{2}$ for our extinction fits; see Section 3.3.1) and bin the flux in blocks of $200 \AA$ (excluding lineaffected regions). Uncertainties are determined by combining the statistical uncertainties from the spectrum with a systematic term of $3 \%$ per bin to incorporate any uncertainty in the flux calibration $(10 \%$ is used for $<5500 \AA$ and $>9000 \AA$, which are especially uncertain.) Using this technique, we generate a line-corrected narrow-band SED spanning 5400-9200 to complement our line-corrected photometry. The afterglow fluxes from direct and synthetic photometry are presented in Tables 3 and 4, respectively.

\subsubsection{Extinction Fitting}

The combined photometric and spectroscopic SED is plotted in Figure 4. It is immediately evident that this curve is unlike almost any other GRB SED that has been observed in detail to date. First, the color is extremely red: a power-law fit to the broadband photometry would give a spectral slope $\left(F \propto v^{-\beta}\right)$ of $\beta \approx 3$, at odds with the theoretically expected value of $\beta=0.5-1.2$ for a fading early afterglow (Sari et al. 1998). Second, it is not monotonic: the flux drops sharply from the $K_{s}$ band until $\sim 2200 \AA$ in the rest frame before actually recovering, showing a local maximum at $\sim 1600 \AA$ before falling again further to the blue.

These properties are immediately recognizable as signatures of dust extinction, and particularly of MW-like extinction with its broad $2175 \AA$ absorption band. This strong extinction imprint, in combination with our high-S/ $\mathrm{N}$ afterglow observations spanning the entire optical/near-IR window, permits analysis of the rest-frame UV extinction properties at a level of detail that 
Table 4

Binned, Line-interpolated Keck Spectroscopy

\begin{tabular}{cc}
\hline \hline$\lambda$ & $\begin{array}{c}F_{v, \text { cont }} \\
(\mu \mathrm{Jy})\end{array}$ \\
\hline 5448.56 & $157.73 \pm 1.15$ \\
5670.14 & $180.13 \pm 2.20$ \\
5842.98 & $197.47 \pm 3.05$ \\
6112.85 & $248.35 \pm 3.11$ \\
6235.50 & $241.48 \pm 2.14$ \\
6476.67 & $260.41 \pm 3.02$ \\
6776.25 & $287.44 \pm 1.82$ \\
7099.05 & $296.41 \pm 2.11$ \\
7281.72 & $293.54 \pm 1.28$ \\
7483.82 & $284.98 \pm 1.19$ \\
7774.03 & $262.52 \pm 2.97$ \\
7997.30 & $240.04 \pm 1.04$ \\
8181.51 & $230.34 \pm 1.04$ \\
8411.10 & $222.31 \pm 2.78$ \\
8607.66 & $203.17 \pm 1.25$ \\
8759.01 & $189.30 \pm 8.11$ \\
8892.83 & $186.93 \pm 5.67$ \\
9044.91 & $200.48 \pm 3.56$ \\
9175.62 & $195.13 \pm 2.90$ \\
\hline & \\
\hline &
\end{tabular}

Notes. Uncertainties are photometric only and do not include any systematic term. Fluxes are corrected for $\mathrm{H}_{2}^{*}$ absorption in the host galaxy and for Galactic extinction.

is almost never possible with GRBs (or, indeed, with any other technique at this redshift range).

To constrain the dust properties, we initially followed the standard procedure (e.g., Jakobsson et al. 2003; Schady et al. 2007; Heng et al. 2008; Kann et al. 2010) for GRB extinction measurements by fitting the average MW, Large Magellanic Cloud (LMC), and Small Magellanic Cloud (SMC) curves, assuming an intrinsic power-law spectrum. (Here, and elsewhere unless otherwise specified, the intrinsic spectral slope over the optical range is fixed at $\beta=0.7$. Fortunately, because the amount of extinction for this burst is so large, deviations from this assumption do not significantly affect our results, except to slightly increase the uncertainties in the derived parameters, as we will discuss in Section 3.4.) In all three cases, we use the Fitzpatrick (1999) parameterization of Local-Group extinction as implemented in the GSFC IDL package, with $R_{V}$ fixed to their average value for each galaxy; for SMC extinction we use the Fitzpatrick parameters from Gordon et al. (2003) (SMC bar average). SMC extinction is ruled out (it converges to $A_{V}=0$ mag with $\chi^{2} /$ dof $=1159 / 24$ ), as it rises steeply to the far-UV (FUV) and does not allow for the $2175 \AA$ bump feature that is so prominent in our data. The LMC and MW curves fit the data much better, but nevertheless they are not statistically acceptable either. Both curves are too flat in the observed IR; the MW curve also significantly overestimates the strength of the $2175 \AA$ bump.

This should not be a surprise: even within our own Galaxy a significant diversity of extinction laws is evident. The majority of observed Galactic sightlines are consistent with variation in a single parameter $R_{V}$, which describes the relative "grayness" (wavelength independence) of the extinction at optical through UV wavelengths (Cardelli et al. 1989; hereafter CCM). A small number of sightlines in the MW (and all sightlines within the LMC and SMC) require additional parameters to fit accurately. A more general Local-Group extinction law,

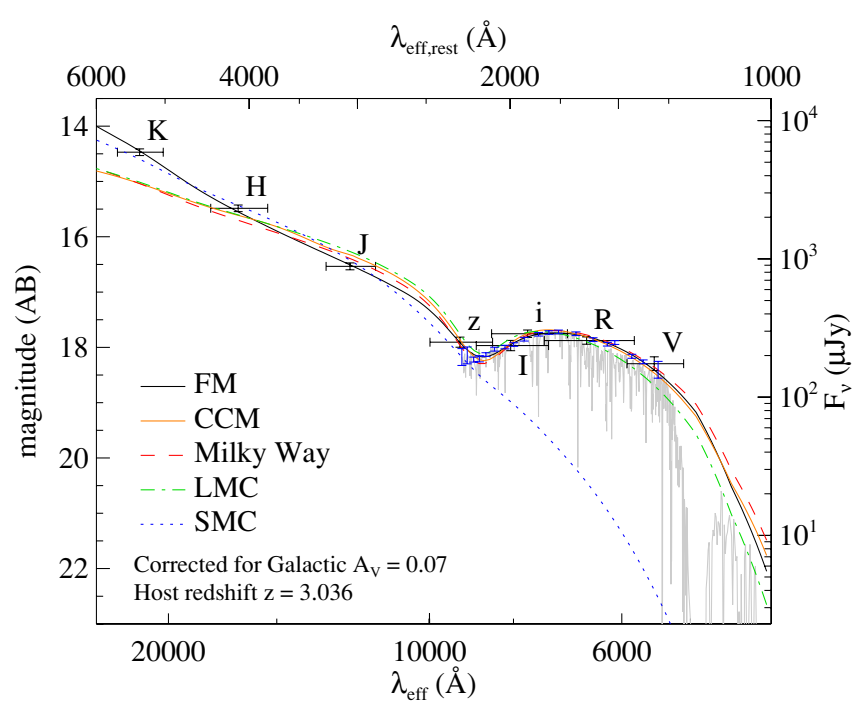

Figure 4. Combined photometric and spectroscopic SED of GRB 080607 fitted with several different extinction models (FM: Fitzpatrick \& Massa 1990 and CCM: Cardelli et al. 1989). Black error bars indicate broadband photometry; blue error bars show the binned pseudo-photometry as derived from the Keck spectrum and corrected for line absorption (including $\mathrm{H}_{2}^{*}$ ). The light gray line shows the spectrum (mostly unbinned and including all lines). Several different extinction fits are shown; only the general FM model (solid black) is an acceptable fit to the data. The SMC curve shown is a fit to the IR data only (an SMC fit to all data converges to $A_{V}=0 \mathrm{mag}$ ).

(A color version of this figure is available in the online journal.)

developed by Fitzpatrick \& Massa (1990; hereafter FM), is able to fit essentially all local sightlines by adding an additional family of parameters: $c_{2}$ for further variations in steepness in the UV, $c_{3}$ for the strength of the $2175 \AA$ bump, $\gamma$ for the bump's width, and $c_{4}$ for the strength of the FUV rise. (The parameter $c_{1}$ is also present in principle, but it is essentially degenerate with $c_{2}$ and $R_{V}$, and in practice it is fixed based on those values. In addition, the parameter $x_{0}$ describes the central wavelength of the $2175 \AA$ bump, but it has not been conclusively shown to vary and is fixed to the average value.)

We first attempted to fit using the general FM law (joined to the standard CCM law in the rest-frame optical with a spline), leaving all parameters free (except $c_{1}$ and $x_{0}$ as described above). Unfortunately, because our observations do not extend far enough into the rest-frame optical to properly constrain the optical/IR extinction properties independent of the UV, the $R_{V}$ parameter is effectively unconstrained in this case. Fortunately, $R_{V}$ and $c_{2}$ also are tightly correlated locally and can be tied together-using, for example, the correlation of Fitzpatrick (1999) (linear) or that of Reichart (2001) (quadratic, allowing for the optically flat, steep-UV SMC-like curve). Both correlations give acceptable (and very similar) fits to our data, and the Fitzpatrick-constrained curve is shown in Figures 4 and 5.

We also attempted a range of non-FM models, such as those of Calzetti et al. (2000), Maiolino et al. (2004), and Gaskell et al. (2004). These curves all lack the $2175 \AA$ bump and do not fit the data well. In addition, we tried to fit the multi-parametric extinction curve from $\mathrm{Li}$ et al. (2008), which can incorporate the $2175 \AA$ bump and gives a fairly reasonable fit (however, the $c_{1}$ parameter diverges and had to be fixed manually, and the result is significantly worse than the FM curve). As the Li curve has not been used extensively on local sightlines, it is difficult to interpret the results, and we will not discuss it further.

The results from our various fits are presented in Table 5. Note that despite the qualitative similarity of the curve to MW 
Table 5

Extinction Fits

\begin{tabular}{lcccccccc}
\hline \hline Model & $\begin{array}{c}A_{V} \\
(\mathrm{mag})\end{array}$ & $R_{V}$ & $c_{1}$ & $c_{2}$ & $c_{3}$ & $c_{4}$ & $\gamma$ & $\chi^{2} / \mathrm{dof}$ \\
\hline Average MW & $1.25 \pm 0.03$ & 3.1 & -0.07 & 0.70 & 3.23 & 0.41 & 0.99 & $127 / 24$ \\
Average LMC & $1.09 \pm 0.02$ & 3.2 & -1.28 & 1.11 & 2.73 & 0.64 & 0.91 & $275 / 24$ \\
LMC2 & $0.16 \pm 0.03$ & 2.6 & -2.16 & 1.31 & 1.92 & 0.42 & 1.05 & $1143 / 24$ \\
SMC & $0 \pm 0.01$ & 2.73 & -4.96 & 2.26 & 0.37 & 0.46 & 0.99 & $1159 / 24$ \\
CCM & $0.82 \pm 0.06$ & $2.41 \pm 0.12$ & & & & & $123 / 22$ \\
FM+tie & $3.26 \pm 0.31$ & $4.17 \pm 0.15$ & $1.11 \pm 0.12$ & $0.31 \pm 0.04$ & $1.70 \pm 0.29$ & $0.28 \pm 0.07$ & $1.10 \pm 0.06$ & $24.2 / 20$ \\
FM+Reichart & $3.52 \pm 0.35$ & $4.69 \pm 0.19$ & $1.29 \pm 0.15$ & $0.30 \pm 0.05$ & $1.66 \pm 0.30$ & $0.31 \pm 0.07$ & $1.07 \pm 0.07$ & $22.9 / 20$ \\
Li & $1.70 \pm 0.06$ & & 200 & $12.3 \pm 0.6$ & $14 \pm 285$ & $0.03 \pm 0.01$ & $38.7 / 20$ \\
\hline
\end{tabular}

Notes. Comparison of fits to the SED of GRB 080607 using a variety of extinction models, most of which cannot adequately fit the observations. Because the optical spectrum and photometry dominate the observations, most models converge to a low-extinction value to try to accommodate the weak $2175 \AA ̊$ bump and seemingly flat spectrum. These models are not consistent with the red IR color. Both a high $R_{V}$ and a low $c_{3}$ are required to explain the optical and IR data together, as reflected in the FM fits. Parameter uncertainties do not include the effect of the uncertain intrinsic spectral index $\beta$ (a value of 0.7 is assumed).

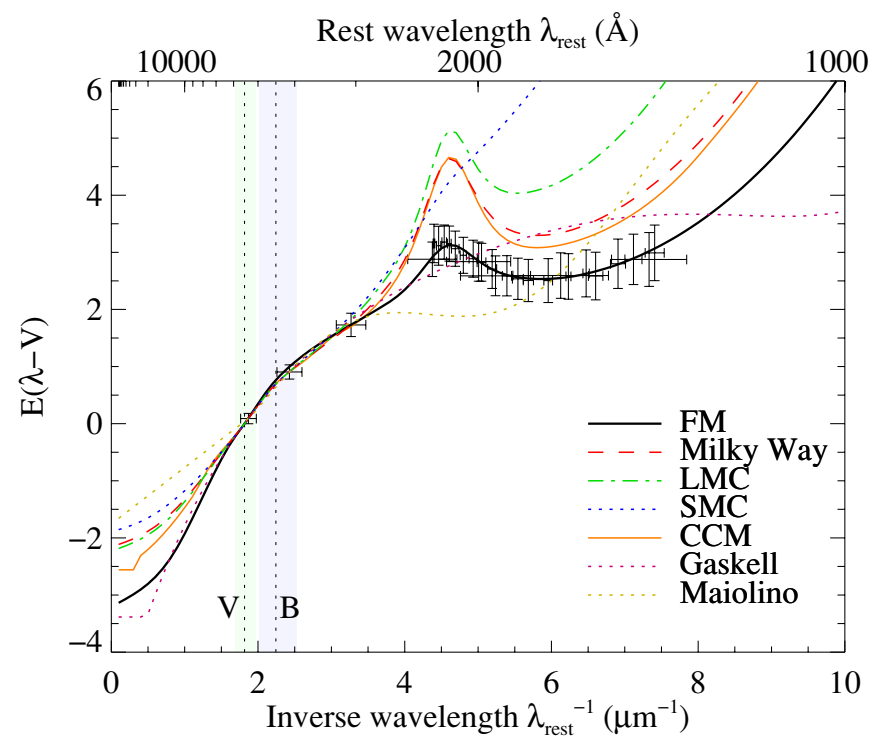

Figure 5. Different extinction curves compared with our afterglow data, shown as the selective extinction $E(\lambda-V)=A_{\lambda}-A_{\mathrm{V}}$. All curves are normalized to match the observed $B-V$ color (traditionally, UV extinction curves are plotted as $E(\lambda-V) / E(B-V))$. This illustrates the flatter nature of the derived extinction curve (higher $R_{V}$ ) and weaker $2175 \AA$ bump required along the GRB sightline relative to the average MW or LMC sightlines.

(A color version of this figure is available in the online journal.)

and LMC sightlines, three major parameters $\left(R_{V}=4.17 \pm 0.15\right.$, $c_{3}=1.70 \pm 0.29$, and $\left.c_{4}=0.28 \pm 0.07\right)$ differ significantly from the average MW and LMC values ( $\gamma$ is consistent with the average MW value). In general, the GRB 080607 sightline is UV-greyer, and its $2175 \AA$ bump weaker, than the average MW sightline. Still, all these properties are in the range seen along different sightlines locally (e.g., from Fitzpatrick \& Massa 1990: $\left.2.3<R_{V}<6.6,1.2<c_{3}<4.5,0.15<c_{4}<0.90\right)$. No single local analog appears to match the properties seen toward the GRB exactly, but it is nevertheless notable that our data are so well fitted by the standard, locally derived laws without the need for any unusual parameters. ${ }^{14}$ We will further discuss the implications of the FM parameters in Section 4.3.

\footnotetext{
14 This is not simply a matter of the flexibility of the fitting function: the model is quite limited in scope, with only four free parameters, each of which is constrained to a small allowable range. Indeed, some reported extragalactic sightlines, e.g., the high- $z$ QSO sightline of Maiolino et al. (2004), cannot be accurately fitted within this model.
}

Assuming any particular extinction model is not strictly necessary for this GRB: the large extinction column actually allows us to directly measure the wavelength-dependent extinction without need for fitting. Traditionally, UV extinction curves are presented as $E(\lambda-V)$ (i.e., $A_{\lambda}-A_{V}$; the optical extinction itself $A_{V}$ need not be known). Our $K_{s}$-band measurement corresponds to the rest-frame $V$ band, and so if the intrinsic slope can be assumed, one can simply measure this value for each filter (or wavelength bin) by comparing the observed $\lambda-V$ color to the predicted color for the assumed intrinsic spectrum. The results are plotted in Figure 5, illustrating the intrinsic differences between the curves and the inability of most of them to fit the data.

\subsection{Effect of Varying Intrinsic $\beta$}

The above quoted results all assume $\beta=0.7$. In reality, we do not know the exact intrinsic spectral index, which varies from burst to burst. ${ }^{15}$ As previously mentioned, the extinction of this burst is sufficiently large, and the intrinsic variation in $\beta$ between events relatively small, that the errors introduced from variation in the spectral index are small. Here, we quantify that statement and propagate the effects into our parameter uncertainties.

Kann et al. (2010) have compiled photometry for a large number of bright, well-observed, Swift-era GRBs and performed fits to the extinction (using the standard MW/LMC/SMC method) and spectral index of each event. We downloaded the data in Table 2 of that work and removed all events which did not have a best-fit (among the three models) $A_{V}<$ $0.2 \mathrm{mag}$ within $2 \sigma$ to exclude events with significant or poorly determined extinction. We further removed any events reporting an unphysical $A_{V}<0$ mag at more than $2 \sigma$ and any event with an uncertainty in its derived spectral index $\sigma_{\beta}>0.2$. The intrinsic spectral indices of this final sample of 21 lowextinction, well-constrained bursts have an average spectral index of $\beta=0.70$ and standard deviation $\sigma_{\beta}=0.26$. We take

\footnotetext{
15 In theory, closure relations (e.g., Price et al. 2002) allow $\beta$ to be calculated from the light-curve decay slope $\alpha$. However, as discussed in Section 4.2, we are unable to unambiguously determine whether the burst medium is in an interstellar medium or wind environment, or whether the early observations are reverse-shock dominated, so it is not clear which relation is most appropriate. Furthermore, even for bursts where no extinction is present and $\beta$ can be measured directly, closure relations sometimes fail to accurately relate the observed parameters (e.g., Racusin et al. 2009). To avoid dependency of our conclusions on the details of the uncertain early-time afterglow physics, we therefore adopt the entirely empirical treatment of $\beta$ described in this section.
} 
Table 6

FM Extinction Parameters for GRB 080607

\begin{tabular}{lcc}
\hline \hline Parameter & $\begin{array}{c}\text { Optical Alone } \\
\text { Value }\end{array}$ & $\begin{array}{c}\text { Optical + X-ray } \\
\text { Value }\end{array}$ \\
\hline$\beta$ & $0.70 \pm 0.26$ & $1.08 \pm 0.05$ \\
\hline$E_{B-V}$ & $0.78 \pm 0.09$ & $0.68 \pm 0.04$ \\
$A_{V}$ & $3.26 \pm 0.35$ & $3.07 \pm 0.32$ \\
$R_{V}$ & $4.17 \pm 0.25$ & $4.52 \pm 0.23$ \\
$c_{1}$ & $1.11 \pm 0.20$ & $1.37 \pm 0.15$ \\
$c_{2}$ & $0.31 \pm 0.07$ & $0.22 \pm 0.05$ \\
$c_{3}$ & $1.70 \pm 0.30$ & $1.82 \pm 0.32$ \\
$c_{4}$ & $0.28 \pm 0.08$ & $0.37 \pm 0.08$ \\
$\gamma$ & $1.10 \pm 0.07$ & $1.07 \pm 0.06$ \\
$x_{0}$ & 4.596 & 4.596 \\
\hline
\end{tabular}

Notes. Final FM extinction parameters for GRB 080607. The values in the left column incorporate only the optical data and include the effect of unknown intrinsic spectral index. Values at right assume an unbroken power law between the optical and X-rays. The values of $c_{1}$ and $R_{V}$ are tied to $c_{2}$ as described in the text; the best-fit value of $A_{V}$ is likewise dependent on this tie, although all other parameters are independent of $R_{V}$. The resulting $R_{V}$ is significantly higher (i.e., $c_{2}$ is lower) than the average MW or LMC curves but has a typical value for dense sightlines. The $2175 \AA$ bump (strength given by $c_{3}$ ), ubiquitous in the MW but nearly absent in the SMC, is present but weaker than in the MW or LMC.

this as a representative sample with which to determine a prior on the intrinsic (unextinguished) spectral index $\beta$.

The observed spectral index between the $J$ and $K_{s}$ bands for this GRB is $\beta=3.5$, so the impact of reddening (between these wavelengths) from dust is clearly much larger (by about an order of magnitude) than the typical variation in the intrinsic spectral index. This variation in the intrinsic index is, however, the largest source of uncertainty in the measurement of the extinction parameters. To take this into account, we refitted our preferred extinction models for the $\pm 1 \sigma$ cases and combined the resulting variation of the best-fit value in quadrature with the statistical uncertainties on the $\beta=0.7$ fit. The final values for all extinction parameters (using the Fitzpatrick $c_{2}-R_{V}$ correlation; the Reichart correlation is not significantly different) are presented in Table 6.

As an alternative to assuming an intrinsic optical $\beta$, we also attempted our fits by including the $\mathrm{X}$-ray flux value at the extraction epoch and assuming an unbroken power law over the full range between the optical and X-ray data (which allows for a much more precise derivation of $\beta$ as well as a constraint on the overall flux normalization, though it is strongly dependent on this assumption of an unbroken intrinsic index). This gives generally quite consistent values with our optical-only fit, in further support of our assertion that the derived dust properties are not strongly affected by our assumptions about the intrinsic spectrum.

\subsection{X-ray Scattering?}

Of particular note in Table 6 , and consistent with our previous work (Prochaska et al. 2009), is the conclusion of a large extinction column $\left(A_{V}=3.26 \pm 0.35 \mathrm{mag}\right)$. This identification of GRB 080607 as a highly extinguished event makes it a potentially useful test case of the X-ray scattering model for early-time afterglows (Shen et al. 2009). However, even $A_{V}=$ $3 \mathrm{mag}$ is generally inadequate to expect any significant effects on the X-ray light curve in this case. Following the discussion by Shen et al. (2009), we calculate the $1 \mathrm{keV}$ specific fluence from the prompt emission using the parameters given by Golenetskii et al. (2008) and integrate the X-ray afterglow flux (starting at $100 \mathrm{~s}$, and ignoring the X-ray flare) using our power-law fit. The resulting ratio of $S_{\mathrm{AG}} / S_{\text {prompt }}=\left(1.0 \times 10^{-6}\right) /\left(1.6 \times 10^{-7}\right)=$ 6.3 places an upper limit on the scattering opacity at this wavelength (i.e., $\tau_{\text {scat }}<6.3$ ). Translating this to a limit on the optical opacity using Equation (8) of Shen et al. (2009), the limiting dust extinction for this case is the thoroughly unconstraining $A_{V}<686 \mathrm{mag}$, a value about 200 times higher than our direct measurement. Equivalently, the total fluence of the dust-scattered X-rays for this event is anticipated to be 200 times lower than the actual afterglow fluence observed, and therefore undetectable. Indeed, for this event, the X-ray light curve follows a simple unbroken power law and (with the exception of the early X-ray flare, a prompt-emission feature; Kocevski et al. 2007; Chincarini et al. 2007) no significant hardness variations of the type predicted by Shen et al. (2009). We conclude that, despite the large surrounding dust column, $\mathrm{X}$-ray scattering is not significant for this GRB.

\section{DISCUSSION}

\subsection{Afterglow Luminosity in Context}

The impressive optical brightness for an event at $z=3$ has already been noted. In fact, as we shall show, after the effects of extinction are taken into account, GRB 080607 is among the most optically luminous GRBs (and therefore objects of any sort) to date, second only to GRB 080319B.

Following Kann et al. (2007, 2010), we select as our comparison filter the $z=1 R$ band (that is, the wavelength which is shifted to the observed $R$ band if at $z=1$ ); this corresponds roughly to the rest-frame $U$ band. For GRB 080607, this is shifted all the way to approximately the observed $J$ band. Therefore, taking advantage of the apparent lack of color change, we shift all other filters to the $J$-band light curve using our model fluxes, extending this curve back to the observed emission peak. This curve is corrected for Galactic extinction (only $0.02 \mathrm{mag}$ ), for host extinction (4.94 mag), and for the difference in luminosity distance between $z=1$ and $z=3.036$ using standard cosmological parameters $\left(h=0.7, \Omega_{M}=0.3, \Omega_{\Lambda}=0.7\right)$. A small $K$-correction is then applied to match the spectrum exactly with the $z=1 R$ band, and the light curve is scaled (undilated) to $z=1$.

The result is plotted in Figure 6, compared with the light curves of GRB 080319B and the three next most luminous events (from Figure 7 of Bloom et al. 2009), and with the peak luminosities of a large sample of well-studied Swift bursts (from Figure 7 of Kann et al. 2010). At the beginning of observations, GRB 080607 is comparable in luminosity to GRB 080319B (and at early times was likely brighter), but the prompt optical flaring of GRB 080319B pushes that burst to a higher luminosity over the next several minutes. GRB 080607 remains among the five most luminous bursts for the rest of its observed evolution. This illustrates the remarkable attributes of this burst that allowed it to provide such a detailed analysis of its environment. In terms of the afterglow (external-shock) emission alone, GRB 080607 may yet be the most luminous: the peak of GRB 080319B appears to correlate with its prompt emission and fades particularly rapidly when the prompt emission ends; the origin of its early-time optical emission is still debated (Racusin et al. 2008). The optical light curve of GRB 080607 bears no relation to the prompt emission and is certainly external-shock dominated at all times. 


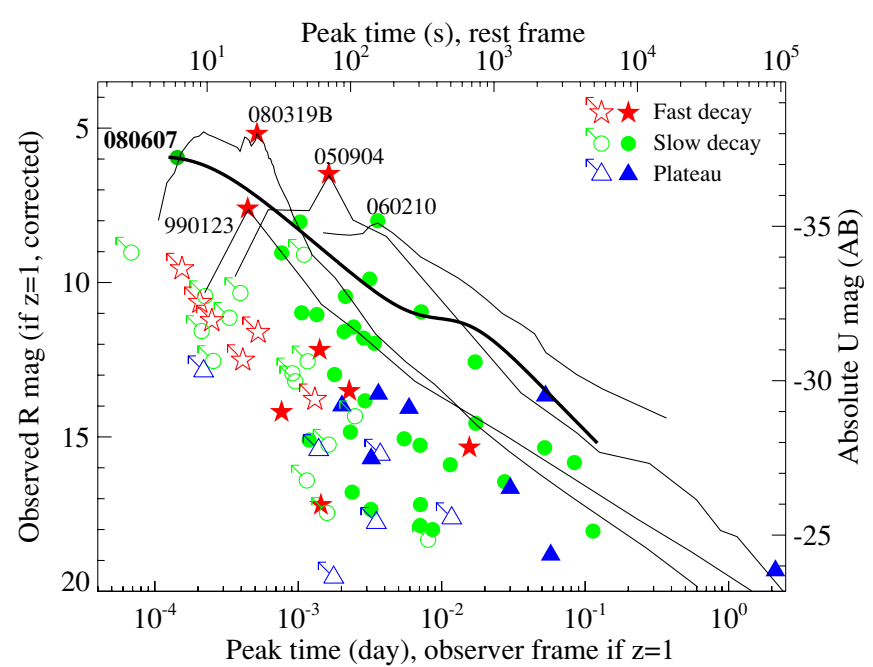

Figure 6. Near-UV luminosity of GRB 080607 (bold curve) compared to several other prominent bursts, as well as to a large sample of rapidly observed Swift GRBs from Kann et al. (2010). Colored points indicate peak observed luminosities of the events described in that paper (unfilled points are events caught after the peak and therefore only lower limits on the peak luminosity). At peak, GRB 080607 is among the most luminous GRB known, peaking at $M_{U} \approx-37 \mathrm{mag}$. At $z=1$, it would peak at mag 6 if unobscured by dust.

(A color version of this figure is available in the online journal.)

\subsection{Physical Properties}

Unfortunately, the physical properties responsible for making GRB 080607 so energetic remain mostly hidden from view. In the burst rest frame, our optical observations extend only to $10^{3} \mathrm{~s}$ and the X-ray observations cease at $t \approx 1$ day, which does not usefully constrain the jet opening angle. Conservatively setting $t_{\text {jet }}>6 \times 10^{4} \mathrm{~s}$ using the X-ray light curve, following the standard equations for the jetting time (Sari \& Piran 1999; Frail et al. 2001) and fiducial values of density ${ }^{16} n=100 \mathrm{~cm}^{-3}$ and efficiency $\eta=0.2$, we measure a jet opening angle of $\theta_{\text {jet }}>$ $3.6 \eta_{0.2}^{1 / 8} n_{100}^{1 / 8}$ degrees; the equivalent lower limit on the beamingcorrected gamma-ray energy release is $E_{\gamma}>1.8 \times 10^{51} \eta_{0.2}^{1 / 4} n_{100}^{1 / 4}$ erg, a fairly typical value. It is therefore not clear whether the extreme apparent luminosity of this burst is attributable to intrinsically large energetics (Cenko et al. 2010a), favorable viewing angle (of a nonuniform, centrally concentrated jet, as was suggested for GRB 080319B by Racusin et al. 2008), an intrinsically narrowly concentrated (uniform) jet, or some combination of these parameters.

To a large extent, the optical luminosity is simply another reflection of the total energetics of the burst itself: both in theory (Sari et al. 1998) and observation (Gehrels et al. 2008; Nysewander et al. 2009; Kann et al. 2010), the inferred afterglow luminosity scales approximately linearly with $E_{\text {iso }}$, and if the optical light curve is extrapolated to late times the predicted optical flux is in the middle of the fluence-normalized distribution. However, there is more to the story: the X-ray light curve of this burst (Figure 7) is not (except at the earliest times) particularly bright; when normalized to the burst fluence it is quite typical for a Swift burst at early times (and actually is unusually faint at late times, due to its rapid unbroken decay).

16 This is an unusually large value of $n$, motivated by the apparent low value of the cooling break $v_{c}$ and inference of a dense molecular environment along the line of sight, as discussed later in this section. Fortunately, the value of $n$ only weakly affects the derived value of $\theta$ and $E_{\gamma}$.

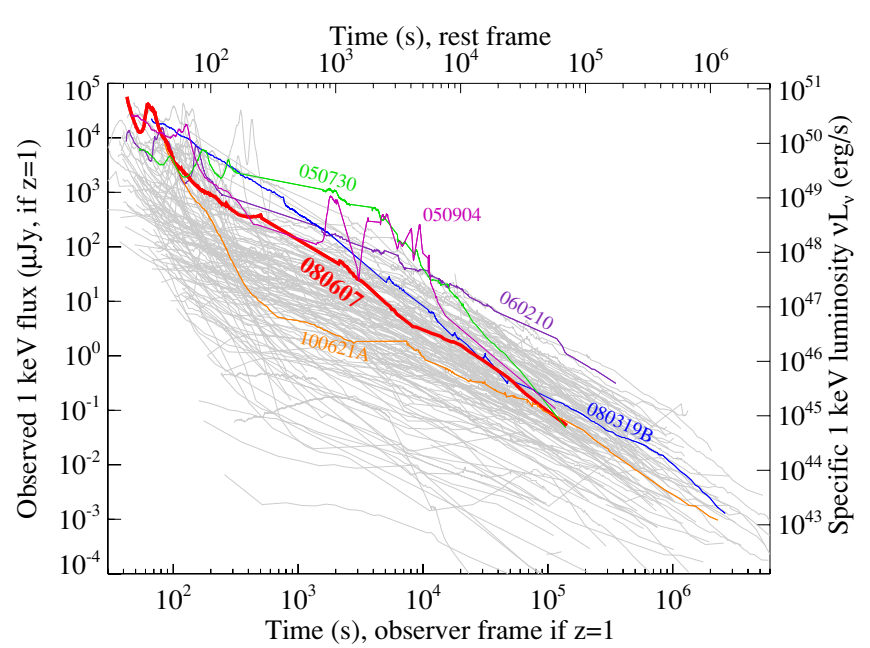

Figure 7. X-ray luminosity of GRB 080607 (bold red curve) compared to all other Swift-followed GRBs. Several other prominent bursts are also individually colored. GRB 080607 is among the most X-ray luminous bursts at peak, but fades quickly to an average luminosity by later times.

(A color version of this figure is available in the online journal.)

There are two broad ways to interpret this. The simplest interpretation is that the cooling-break frequency $v_{c}$ has a particularly low value compared to most GRBs, perhaps even below the optical band (the available data are marginally consistent with the X-ray and optical bands being on a single spectral power law). The obvious culprit for this involves the external density $n$ : the X-ray flux should be independent of density (assuming that $v_{\mathrm{X}}>v_{c}$ for most bursts), but the cooling break and optical flux are sensitive to it $\left(v_{c} \propto n^{-1}\right.$; below the cooling break $F \propto n^{1 / 2}$ ). An external density 10 or 100 times the "typical" Swift value would push the cooling break from its typical position between the optical and X-ray bands into or below the optical band at early times, increasing the optical luminosity. Indeed, after correcting for extinction the early-time broadband SED appears to demand a low cooling-break frequency: the optical-to-X-ray index at only $300 \mathrm{~s}$ is $\beta_{\mathrm{OX}}=1.1$, consistent with the $\mathrm{X}$-ray spectral slope $\left(\beta_{\mathrm{X}}=1.16 \pm 0.13\right)$. The probable low value of the cooling break also helps explain why a similar extinction column is derived whether the optical data are considered alone (the most general case) or in conjunction with the X-ray data assuming an unbroken power law (which requires $v_{c}<v_{\text {opt }}$ ), as demonstrated in Section 3.4. Unfortunately, the period of simultaneous temporal coverage between the optical and X-ray observations is too short to determine, via the light curve, whether a break is present between the bands. (The burst could also have exploded into a wind-stratified medium - one with variable density $n \propto r^{-2}$-in which case $v_{c}$ rises with time and the optical flux fades more rapidly than the $\mathrm{X}$-ray flux, as is observed.)

The alternative interpretation is that the optical flux originates from a separate emission component, the most obvious candidate being the reverse shock (Mészáros \& Rees 1997). Several previous early fast-fading light curves have been associated with reverse shocks (e.g., Akerlof et al. 1999; Kobayashi \& Zhang 2003; Perley et al. 2008; Steele et al. 2009); qualitatively, the behavior of GRB 080607 appears similar to these events, although the decay is somewhat slower and there are no late-time observations to determine whether the light curve became forward-shock dominated as predicted. The factors determining the luminosity of the reverse shock (Zhang et al. 

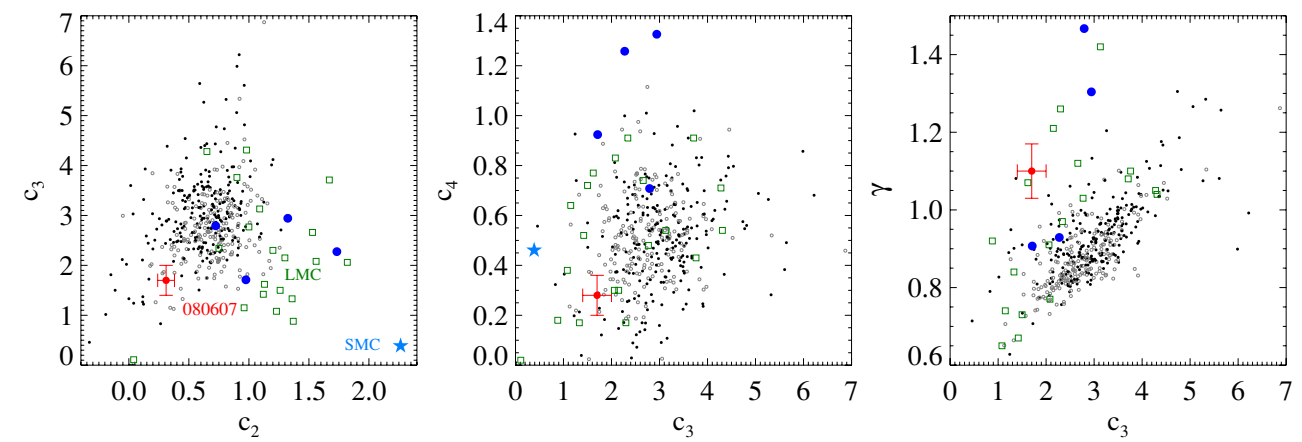

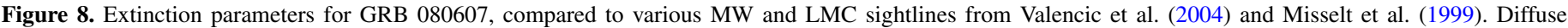

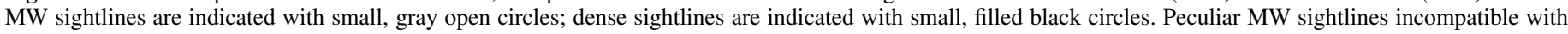

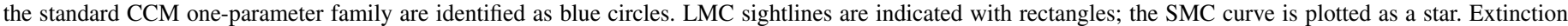

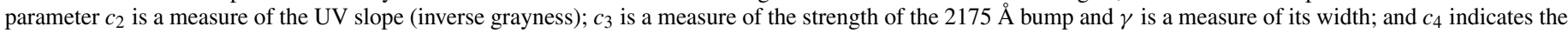

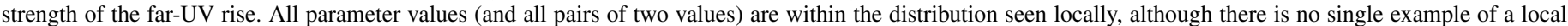
sightline that is consistent with the extinction properties of the GRB 080607 sightline in all aspects.

(A color version of this figure is available in the online journal.)

2003) are generally the same as for the forward shock (and so a high external density would similarly aid the production of a luminous afterglow), but can be further amplified if the magnetization $R_{B}=\epsilon_{B, r} / \epsilon_{B, f}$ of the reverse shock is high, due (for instance) to primordial fields in the ejecta (Gomboc et al. 2008).

Unfortunately, the lack of late-time observations (to search for the appearance of a forward shock) or radio data (to more directly constrain $n$ ) prevents us from distinguishing between these possibilities. Fortunately, we can speak more confidently about the other aspect of this burst's remarkable luminosity: the fact that it peaked so early (even if two bursts have similar energetics and late-time luminosities, the power-law nature of GRB light curves ensures that the event with the earlier peak time will have significantly larger peak brightness, fleeting as it is).

The peak time (for $v>v_{m}$ ) is set by the deceleration timescale of the ejecta (Sari \& Piran 1999). Because the afterglow has already peaked and is fading at the start of our observations, the ejecta must have accumulated enough circumstellar matter to begin to decelerate and develop an external shock by this time: a mere $24 \mathrm{~s}$ after the BAT trigger ( $6 \mathrm{~s}$ in the rest frame), or more conservatively $32 \mathrm{~s}$ after the beginning of the prompt emission ( $8 \mathrm{~s}$ in the rest frame).

Such rapid deceleration generally requires a high initial Lorentz factor $\Gamma$, although a very high interstellar density also contributes. Using Equation (3) of Rykoff et al. (2009), ${ }^{17}$ we estimate $\Gamma>660 \eta_{0.2}^{1 / 8} n_{100}^{-1 / 8}$, where $\eta_{0.2}$ and $n_{100}$ indicate values of the efficiency and external density relative to fiducial values of 0.2 and $100 \mathrm{~cm}^{-3}$, respectively (see also Molinari et al. 2007). Based on the preceding discussion of the late-time optical luminosity, we have chosen an unusually large value for the interstellar density; even in this case the constraint on $\Gamma$ is at the top end of the afterglow-inferred range (if still somewhat below the pair-opacity limits recently provided by the FermiLAT; Abdo et al. 2009b). It is notable that both $E_{\text {iso }}$ and $\Gamma$ are exceptionally large for this burst, which could suggest that the properties may be correlated.

\footnotetext{
17 This equation is strictly valid only for the thin-shell scenario in which the burst duration is less than the deceleration time (Sari \& Piran 1999; Mészáros 2006). This is not strictly true for this GRB, as prompt emission is observed to continue during the light-curve decline. However, as noted previously, the energetics are dominated by a single, bright pulse which ends well before the start of optical observations.
}

\subsection{X-Ray and Optical Properties: the Environment of GRB 080607}

The derivation of precise values for the extinction parameters along the GRB 080607 sightline (Table 5) gives us an additional means for learning about its host environment. Although the reason for the variation of these parameters is not well understood even within the MW, some broad conclusions can be drawn.

First, we note the high value of $R_{V} \approx 4$ (or equivalently, since the parameters are tied in our modeling, the small value of $c_{2}$ ), indicating a relatively flat extinction curve. In the diffuse interstellar medium (in the MW and in other galaxies as well), $R_{V}$ typically takes on lower values of 2-4. UV-flat extinction curves are generally restricted to denser sightlines, probably because grains are able to coagulate to larger sizes (Valencic et al. 2004). (However, dense regions can exhibit low values of $R_{V}$ as well as high values.) The high $R_{V}$ value is therefore suggestive of a dense environment-fully consistent with the conclusion from the atomic and molecular analysis that the sightline penetrates through a dark molecular cloud in its host.

Second, the value of $c_{3}$ is nonzero, indicating a significant $2175 \AA$ absorption bump. This is one of only a few clear detections of this feature at cosmological distances (Motta et al. 2002; Junkkarinen et al. 2004; Ellison et al. 2006; Srianand et al. 2008; Krühler et al. 2008; Elíasdóttir et al. 2009; Noterdaeme et al. 2009; Zhou et al. 2010) and the highest-redshift detection of the feature yet. The identity of the carrier is still unknown (although polycyclic aromatic hydrocarbons and graphite are considered promising candidates; see Draine 2003 for a review) and the processes that cause it to be present or absent are similarly not yet certain: an evolved stellar population (Noll et al. 2007), metallicity (Fitzpatrick 2004), the strength of the UV radiation field (e.g., Gordon et al. 1997), and disturbance of the environment due to shocks (Seab \& Shull 1983) have all been cited in explaining its absence. Generically, however, it seems to be present in almost all sightlines in the MW and LMC, and in nearby disk galaxies, but absent in more disturbed locations such as the SMC, nearby starburst galaxies (Gordon 2005), and at least one highly disturbed sightline within the MW (Valencic et al. 2003). This suggests that the interstellar medium of the host of GRB 080607 is a closer analog of the more quiescent environments found in the MW and LMC than of the extreme conditions of nearby galaxies having high specific star formation rates. The determination of significant pre-existing stellar mass 
and relatively modest specific star formation rate in our parallel study of the host galaxy (Chen et al. 2010) is in agreement with this expectation.

The strength of the bump is, however, weaker than in almost any sightline in either the MW or LMC (Figure 8). Furthermore, the degree to which the bump is weaker does not follow the local correlations: in the MW, very low values of $c_{3}$ tend to correlate with very low values of $c_{2}$ (weakly) and $\gamma$ (strongly). In our case, a low $c_{2}$ is observed, but it is still much higher than for the Orion Nebula sightlines in which the lowest values of $c_{3}$ are seen. This may be an indicator that a different phenomenon is suppressing this carrier than is in operation within the MW Galaxy. Metallicity is not likely the culprit: the molecular cloud giving rise to the observed extinction has near-solar metallicity despite being at $z>3$ (Prochaska et al. 2009).

The strength of the FUV rise, $c_{4}$, is fairly typical for local sightlines. However, the origin of the rise is even less secure than that of the $2175 \AA$ bump and does not significantly constrain the environment.

The X-ray inferred host-galaxy equivalent hydrogen column of GRB 080607 was measured to be $N_{\mathrm{H}}=2.7_{-0.7}^{+0.8} \times 10^{22} \mathrm{~cm}^{-2}$, which is comparable to the neutral hydrogen column $N_{\mathrm{HI}}=$ $1.5_{-0.5}^{+0.6} \times 10^{22}$ derived from the damped Ly $\alpha$ line (Prochaska et al. 2009). This is a very large value, even considering the high extinction in this direction: the ratio of $N_{\mathrm{H}} / A_{V}=$ $8 \times 10^{21} \mathrm{~cm}^{-2} \mathrm{mag}^{-1}$ is several times larger than observed in the MW, although quite typical of GRBs for which both values have been securely measured (e.g., Schady et al. 2010). It is possible that this arises for reasons unrelated to the molecular cloud-for example, if additional dust-free gas is located closer or further along the sightline relative to the molecular cloud that is responsible for the absorption. If intrinsic, this combination of a weak (but present) bump and a large $N_{\mathrm{H}} / A_{V}$ ratio is consistent with the correlation discussed by Elíasdóttir et al. (2009) and Gordon et al. (2003).

\section{CONCLUSIONS}

One of the brightest and best-studied GRBs (at early times) of the Swift era, GRB 080607 holds particular potential for revealing the nature of GRBs and their environments at high redshift. While the relatively limited observed temporal range restricts our ability to study the intrinsic nature of this event, this is more than compensated by the abundant early-time optical/IR data that reveal the detailed properties of the dark-cloud sightline in its distant host.

The utility of this event is perhaps most evident in the context of the class of "dark" GRBs. Many factors, both intrinsic (high $E_{\mathrm{iso}}, \Gamma$, and $n$ ) and extrinsic (large but not extremely large $A_{V}$, a redshift placing the $2175 \AA$ bump in the optical window, and the fortuitous ability to observe immediately with telescopes in both the continental US and Hawaii) had to conspire together to allow the dust properties of this GRB to be observable in such rich detail. Had this event been slightly less luminous ("only" comparable to GRB 990123, 2 mag fainter at most epochs), its afterglow would have been only marginally detected, and only at the earliest times; further decrease in luminosity would have rendered it undetectable with small telescopes. Even a modest increase in the amount of extinction (higher by $A_{V} \approx$ 1-2 mag) or the presence of relatively UV-opaque SMC-like dust would have a similar impact, suppressing all of the optical measurements.

The literature contains many examples of such sources: GRBs with a large dust column but insufficient luminosity to shine through it. Some prominent cases include GRBs 970828 (Djorgovski et al. 2001), 060923A (Tanvir et al. 2008a), 061222A and 070521 (Cenko et al. 2009; Perley et al. 2009), 070306 (Jaunsen et al. 2008), 081221 (Tanvir et al. 2008b), and 090709A (Cenko et al. 2010b). But even these objects were unusually bright or had particularly rapid or deep observations in their favor. A truly typical-luminosity Swift event without rapid or deep observations would completely escape notice in most cases, permitting only shallow limits on its extinction column. Therefore, there is every reason to think that very dusty environments like that of GRB 080607 are actually not uncommon among GRBs (if not necessarily ubiquitous). This is in agreement with our afterglow plus host survey with the P60 and Keck telescopes (Cenko et al. 2009; Perley et al. 2009).

The extinction curve along the GRB 080607 sightline-a dark molecular cloud at $z=3.036$-is quite similar to that of our own Galaxy (with a significant $2175 \AA$ bump), though there are differences in finer details. The success in modeling the extinction curve of this event within entirely locally developed models is in some ways heartening, giving us confidence that with sufficient knowledge we should be able to understand the absorption properties even out to these immense distances. As perhaps the most detailed determination of the extinction properties of a galaxy at cosmological redshift to date, we suggest that the extinction curve in this work may be of use to others attempting to take into account the effects of dust extinction in other galaxies at high redshift (see Appendix). At the same time, some other GRBs and other techniques have also at times pointed to extinction curves that diverge dramatically from local templates, so the topic should continue to be addressed with caution.

Once corrected for extinction, GRB 080607 rivals the "nakedeye burst" GRB 080319B as the most luminous known object in the universe. This extreme early luminosity of GRB 080607 is likely the product of a variety of factors: it has one of the largest $E_{\text {iso }}$ values to date, and its optical luminosity may have been further amplified by a large circumburst density in its host (or, alternatively, a bright reverse shock). Unlike GRB 080319B, GRB 080607 has a smooth optical peak and shows no correlation with prompt emission at that time. Events like GRB 080607 demonstrate the power of GRBs to illuminate the darkest corners of the universe: not just the reionization era (on which much current attention is focused) but also the dustiest regions over the following several billion years when the global star formation rate-much of it occurring behind optically thick dust clouds-was at its maximum. Such dust-obscured regions are extremely difficult to study by other techniques, or even with most GRBs, as demonstrated by the class of "dark" bursts. The combination of early observations and extreme energetics of GRB 080607 were enough to overcome even this difficulty and demonstrate the power of rare, individual events to illuminate these hard-to-study regions and improve our understanding of the early universe.

J.S.B.'s group is partially supported by a grant from the NSF (award 0941742). The Gamma-Ray Afterglows As Probes (GRAASP) collaboration is supported by NASA/Swift Guest Investigator grants NNX08AN90G and NNX09AO99G. A.V.F., S.B.C., and W.L. acknowledge generous financial assistance from Gary \& Cynthia Bengier, the Richard \& Rhoda Goldman Fund, NASA/Swift Grants NNX09AL08G and NNX10AI21G, the TABASGO Foundation, and NSF Grant AST-0908886. 
A.N.M. acknowledges support from an NSF Graduate Research Fellowship.

PAIRITEL is operated by the Smithsonian Astrophysical Observatory (SAO) and was made possible by a grant from the Harvard University Milton Fund, a camera loan from the University of Virginia, and continued support of the SAO and UC Berkeley. The PAIRITEL project is further supported by NASA/Swift Guest Investigator grant NNX08AN84G.

Some of the data presented here were obtained at the W. M. Keck Observatory, which is operated as a scientific partnership among the California Institute of Technology, the University of California, and NASA. The Observatory was made possible by the generous financial support of the W. M. Keck Foundation. We extend special thanks to those of Hawaiian ancestry on whose sacred mountain we are privileged to be guests.

KAIT and its ongoing operation were made possible by donations from Sun Microsystems, Inc., the Hewlett-Packard Company, AutoScope Corporation, Lick Observatory, the NSF, the University of California, the Sylvia \& Jim Katzman Foundation, and the TABASGO Foundation.

This work made use of data supplied by the UK Swift Science Data Centre at the University of Leicester. This research also made use of the NASA/IPAC Extragalactic Database (NED) which is operated by the Jet Propulsion Laboratory, California Institute of Technology, under contract with NASA.

We thank S. Klose for additional computations that confirmed our luminosity results, and we are grateful for the $\mathrm{H}_{2}^{*}$ model developed by B. T. Draine and supplied by Y. Sheffer. We benefited from the excellent assistance of the staffs of the observatories at which we obtained observations. Finally, we acknowledge the hard work and dedication of the Swift team, whose successful mission has made this study possible.

Facilities: Swift, Keck:I (LRIS), FLWO:2MASS, KAIT, PO: $1.5 \mathrm{~m}$

\section{APPENDIX}

\section{APPLYING THE GRB 080607 EXTINCTION LAW}

Because the extinction law we derive for GRB 080607 is contained within the broader Fitzpatrick parameterization, application of this extinction law is relatively straightforward. Using the function $\mathrm{fm}$ _unred.pro in the GSFC IDL library, the extinction law can be calculated using, for example, the following simple code.

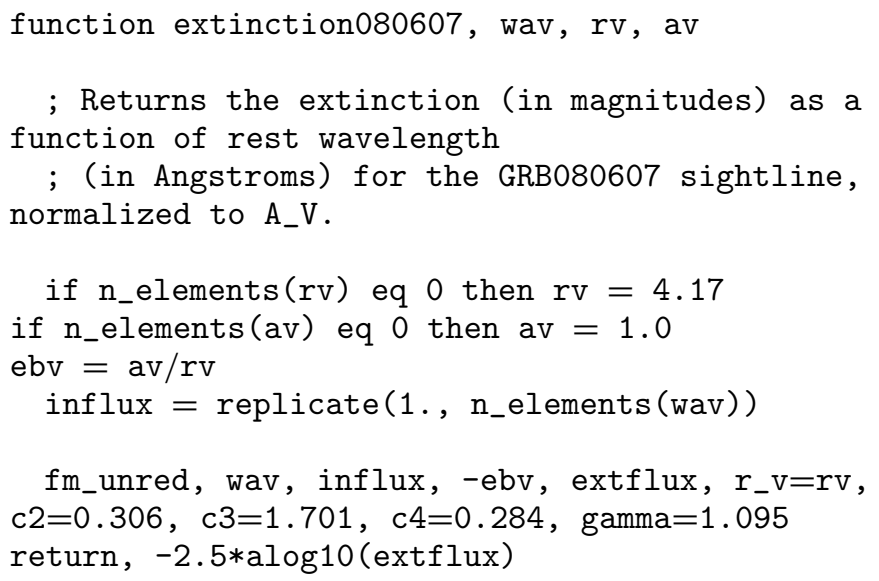

end
The ratio of total to selective extinction, $R_{V}$, is left as a free parameter. The default value of 4.17 is set based on the $R_{V}-c_{2}$ correlation observed in the MW, but other values are allowed ( $R_{V}$ is not directly constrained by the afterglow observations.) The value of $c_{1}$ is tied to $c_{2}$ inside fm_unred.pro.

\section{REFERENCES}

Abazajian, K. N., et al. 2009, ApJS, 182, 543

Abdo, A. A., et al. 2009a, Science, 323, 1688

Abdo, A. A., et al. 2009b, Nature, 462, 331

Akerlof, C. W., et al. 1999, Nature, 398, 400

Akerlof, C. W., et al. 2003, PASP, 115, 132

Band, D., et al. 1993, ApJ, 413, 281

Barthelmy, S. D., Butterworth, P., Cline, T. L., Gehrels, N., Fishman, G. J., Kouveliotou, C., \& Meegan, C. A. 1995, Ap\&SS, 231, 235

Barthelmy, S. D., et al. 2005, Space Sci. Rev., 120, 143

Bertin, E., \& Arnouts, S. 1996, A\&AS, 117, 393

Bertin, E., Mellier, Y., Radovich, M., Missonnier, G., Didelon, P., \& Morin, B. 2002, in ASP Conf. Proc. 281, Astronomical Data Analysis Software and Systems XI, ed. D. A. Bohlender, D. Durand, \& T. H. Handley (San Francisco, CA: ASP), 228

Beskin, G., Karpov, S., Bondar, S., Greco, G., Guarnieri, A., Bartolini, C., \& Piccioni, A. 2010, ApJ, 719, L10

Beuermann, K., et al. 1999, A\&A, 352, L26

Blake, C. H., et al. 2005, Nature, 435, 181

Bloom, J. S., Starr, D. L., Blake, C. H., Skrutskie, M. F., \& Falco, E. E. 2006, in ASP Conf. Ser. 351, Astronomical Data Analysis Software and Systems XV, ed. C. Gabriel, C. Arviset, D. Ponz, \& E. Solano (San Francisco, CA: ASP), 751

Bloom, J. S., et al. 2009, ApJ, 691, 723

Burrows, D. N., et al. 2005, Space Sci. Rev., 120, 165

Butler, N. R., \& Kocevski, D. 2007, ApJ, 663, 407

Butler, N. R., Kocevski, D., Bloom, J. S., \& Curtis, J. L. 2007, ApJ, 671, 656

Calzetti, D., Armus, L., Bohlin, R. C., Kinney, A. L., Koornneef, J., \& StorchiBergmann, T. 2000, ApJ, 533, 682

Cardelli, J. A., Clayton, G. C., \& Mathis, J. S. 1989, ApJ, 345, 245

Cenko, S. B., et al. 2006, PASP, 118, 1396

Cenko, S. B., et al. 2009, ApJ, 693, 1484

Cenko, S. B., et al. 2010a, ApJ, 711, 641

Cenko, S. B., et al. 2010b, AJ, 140, 224

Chen, H.-W., et al. 2010, ApJ, 723, 218

Chincarini, G., et al. 2007, ApJ, 671, 1903

Djorgovski, S. G., Frail, D. A., Kulkarni, S. R., Bloom, J. S., Odewahn, S. C., \& Diercks, A. 2001, ApJ, 562, 654

Draine, B. T. 2003, ARA\&A, 41, 241

Evans, P. A., et al. 2010, A\&A, 519, A102

Ellison, S. L., et al. 2006, MNRAS, 372, L38

Elíasdóttir, Á., et al. 2009, ApJ, 697, 1725

Fitzpatrick, E. L. 1999, PASP, 111, 63

Fitzpatrick, E. L. 2004, in ASP Conf. Ser. 309, Astrophysics of Dust, ed. A. N. Witt, G. C. Clayton, \& B. T. Draine (San Francisco, CA: ASP), 33

Fitzpatrick, E. L., \& Massa, D. 1990, ApJS, 72, 163

Frail, D. A., et al. 2001, ApJ, 562, L55

Gaskell, C. M., Goosmann, R. W., Antonucci, R. R. J., \& Whysong, D. H. 2004, ApJ, 616, 147

Gehrels, N., et al. 2004, ApJ, 611, 1005

Gehrels, N., et al. 2008, ApJ, 689, 1161

Golenetskii, S., et al. 2008, GCN Circ., 7862

Gomboc, A., et al. 2008, ApJ, 687, 443

Gordon, K. D. 2005, in AIP Conf. Proc. 761, The Spectral Energy Distributions of Gas-Rich Galaxies: Confronting Models with Data, ed. C. C. Popescu \& R. J. Tuffs (Melville, NY: AIP), 134

Gordon, K. D., Calzetti, D., \& Witt, A. N. 1997, ApJ, 487, 625

Gordon, K. D., Clayton, G. C., Misselt, K. A., Landolt, A. U., \& Wolff, M. J. 2003, ApJ, 594, 279

Greiner, J., et al. 2009, A\&A, 498, 89

Heng, K., Lazzati, D., Perna, R., Garnavich, P., Noriega-Crespo, A., Bersier, D., Matheson, T., \& Pahre, M. 2008, ApJ, 681, 1116

Jakobsson, P., Hjorth, J., Fynbo, J. P. U., Watson, D., Pedersen, K., Björnsson, G., \& Gorosabel, J. 2004, ApJ, 617, L21

Jakobsson, P., et al. 2003, A\&A, 408, 941

Jaunsen, A. O., et al. 2008, ApJ, 681, 453

Jordi, K., Grebel, E. K., \& Ammon, K. 2006, A\&A, 460, 339

Junkkarinen, V. T., Cohen, R. D., Beaver, E. A., Burbidge, E. M., Lyons, R. W., \& Madejski, G. 2004, ApJ, 614, 658 
Kann, D. A., Masetti, N., \& Klose, S. 2007, AJ, 133, 1187

Kann, D. A., et al. 2010, ApJ, 720, 1513

Kawai, N., et al. 2006, Nature, 440, 184

Kobayashi, S., \& Zhang, B. 2003, ApJ, 582, L75

Kocevski, D., Butler, N., \& Bloom, J. S. 2007, ApJ, 667, 1024

Krühler, T., et al. 2008, ApJ, 685, 376

Kulkarni, S. R., et al. 1998, Nature, 393, 35

Kulkarni, S. R., et al. 1999, Nature, 398, 389

Li, A., Liang, S. L., Kann, D. A., Wei, D. M., Klose, S., \& Wang, Y. J. 2008, ApJ, 685,1046

Li, W., Filippenko, A. V., Chornock, R., \& Jha, S. 2003, PASP, 115, 844

Maiolino, R., Schneider, R., Oliva, E., Bianchi, S., Ferrara, A., Mannucci, F., Pedani, M., \& Roca Sogorb, M. 2004, Nature, 431, 533

Mészáros, P. 2006, Rep. Prog. Phys., 69, 2259

Mészáros, P., \& Rees, M. J. 1997, ApJ, 476, 232

Misselt, K. A., Clayton, G. C., \& Gordon, K. D. 1999, ApJ, 515, 128

Molinari, E., et al. 2007, A\&A, 469, L13

Motta, V., et al. 2002, ApJ, 574, 719

Noll, S., Pierini, D., Pannella, M., \& Savaglio, S. 2007, A\&A, 472, 455

Noterdaeme, P., Ledoux, C., Srianand, R., Petitjean, P., \& Lopez, S. 2009, A\&A, 503,765

Nysewander, M., Fruchter, A. S., \& Pe'er, A. 2009, ApJ, 701, 824

Odewahn, S. C., et al. 1998, ApJ, 509, L5

Oke, J. B., et al. 1995, PASP, 107, 375

Panaitescu, A., Meszaros, P., \& Rees, M. J. 1998, ApJ, 503, 314

Perley, D. A., et al. 2008, ApJ, 672, 449

Perley, D. A., et al. 2009, AJ, 138, 1690

Perley, D. A., et al. 2010, MNRAS, 406, 2473

Price, P. A., et al. 2002, ApJ, 572, L51

Prochaska, J. X., et al. 2009, ApJ, 691, L27

Quimby, R. M., et al. 2006, ApJ, 640, 402

Racusin, J. L., et al. 2008, Nature, 455, 183

Racusin, J. L., et al. 2009, ApJ, 698, 43

Ramaprakash, A. N., et al. 1998, Nature, 393, 43

Reichart, D. E. 2001, ApJ, 553, 235

Roming, P. W. A., et al. 2005, Space Sci. Rev., 120, 95

Rumyantsev, V., \& Pozanenko, A. 2008, GCN Cir., 7891
Rykoff, E. S., et al. 2009, ApJ, 702, 489

Sari, R., \& Piran, T. 1999, ApJ, 520, 641

Sari, R., Piran, T., \& Narayan, R. 1998, ApJ, 497, L17

Schady, P., \& Mangano, V. 2008, GCN Cir., 7858

Schady, P., et al. 2007, MNRAS, 377, 273

Schady, P., et al. 2010, MNRAS, 401, 2773

Seab, C. G., \& Shull, J. M. 1983, ApJ, 275, 652

Sheffer, Y., Prochaska, J. X., Draine, B. T., Perley, D. A., \& Bloom, J. S. 2009, ApJ, 701, L63

Shen, R.-F., Willingale, R., Kumar, P., O'Brien, P. T., \& Evans, P. A. 2009, MNRAS, 393, 598

Skrutskie, M. F., et al. 2006, AJ, 131, 1163

Srianand, R., Gupta, N., Petitjean, P., Noterdaeme, P., \& Saikia, D. J. 2008, MNRAS, 391, L69

Steele, I. A., Mundell, C. G., Smith, R. J., Kobayashi, S., \& Guidorzi, C. 2009, Nature, 462, 767

Stetson, P. B. 1987, PASP, 99, 191

Sugita, S., et al. 2008, in AIP Conf. Ser. 1000, Gamma-ray Bursts 2007, ed. M. Galassi, D. Palmer, \& E. Fenimore (Melville, NY: AIP), 354

Tanvir, N. R., Levan, A. J., Wiersema, K., Fruchter, A. S., \& Graham, J. 2008a, GCN Cir., 8698

Tanvir, N. R., et al. 2008b, MNRAS, 388, 1743

Valencic, L. A., Clayton, G. C., \& Gordon, K. D. 2004, ApJ, 616, 912

Valencic, L. A., Clayton, G. C., Gordon, K. D., \& Smith, T. L. 2003, ApJ, 598 369

Vestrand, W. T., et al. 2005, Nature, 435, 178

Williams, G. G., Milne, P. A., Park, H. S., Barthelmy, S. D., Hartmann, D. H., Updike, A., \& Hurley, K. 2008, in AIP Conf. Ser. 1000, Gamma-ray Bursts 2007, ed. M. Galassi, D. Palmer, \& E. Fenimore (Melville, NY: AIP), 535

Williams, G. G., et al. 2004, in AIP Conf. Proc. 727, Gamma-Ray Bursts: 30 Years of Discovery, ed. E. E. Fenimore \& M. Galassi (Melville, NY: AIP), 723

Woźniak, P. R., Vestrand, W. T., Panaitescu, A. D., Wren, J. A., Davis, H. R., \& White, R. R. 2009, ApJ, 691, 495

Yost, S. A., et al. 2007, ApJ, 657, 925

Zhang, B., Kobayashi, S., \& Mészáros, P. 2003, ApJ, 595, 950

Zhou, H., Ge, J., Lu, H., Wang, T., Yuan, W., Jiang, P., \& Shan, H. 2010, ApJ, 708,742 